Historic, Archive Document

Do not assume content reflects current scientific knowledge, policies, or practices. 


$$
\text { , }
$$




\subsection{3}

LIBRARY ancrorvers

- JUN $131929 \star$

Q

is

MOHICAN

PEONIES

1929

ORDER BEFORE JUNE 30th

It is often convenient for our customers

to order in the spring for fall ship-

ment and we therefore offer a spe-

cial inducement of a Ten per cent

discount on all orders accom-

panied by remittance and

mailed us not later than

June $30 t h$. This dis-

count is given in ad-

dition to the quan-

tity discounts

on page 3. 


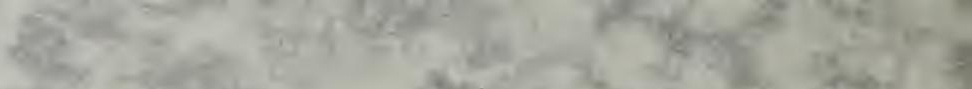

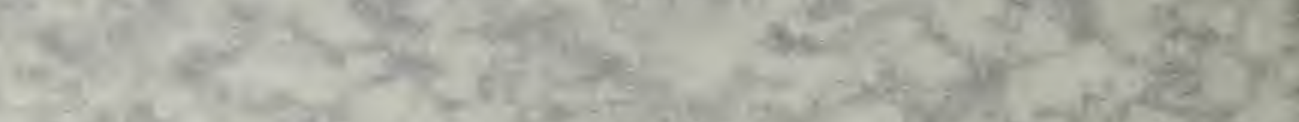

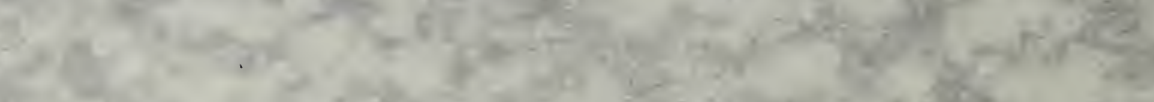

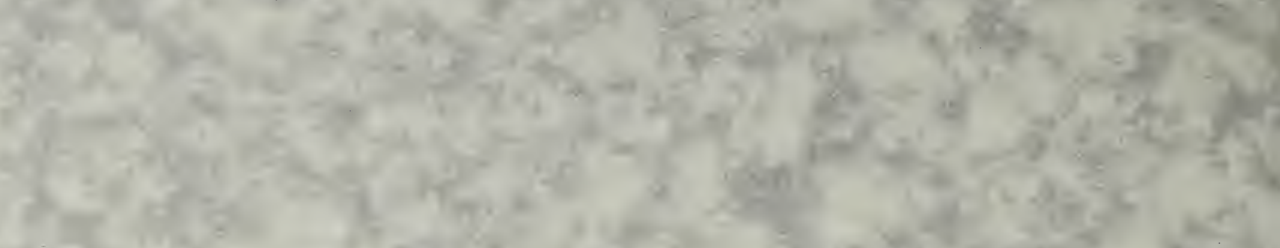
-

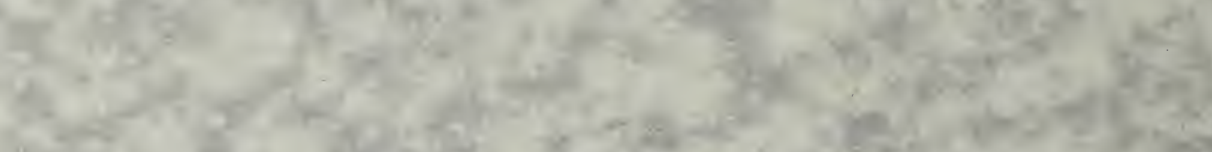

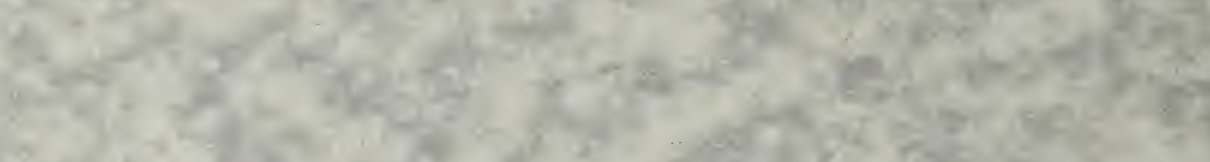
-

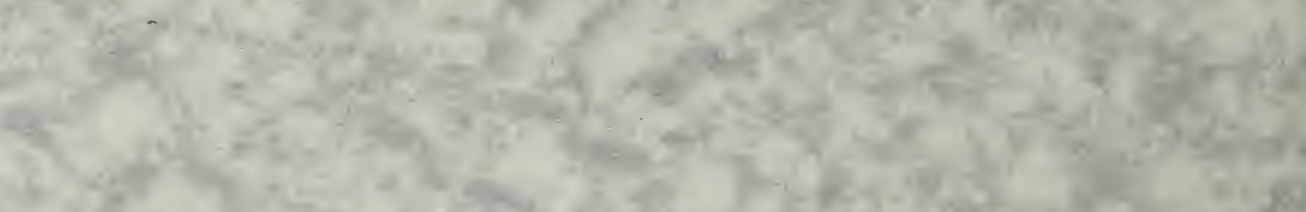

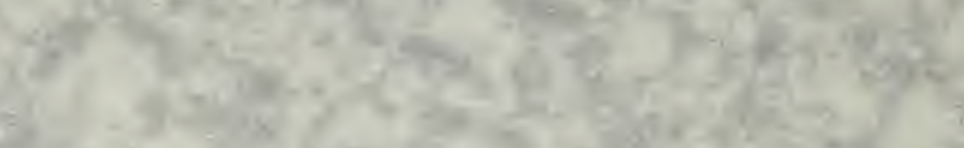

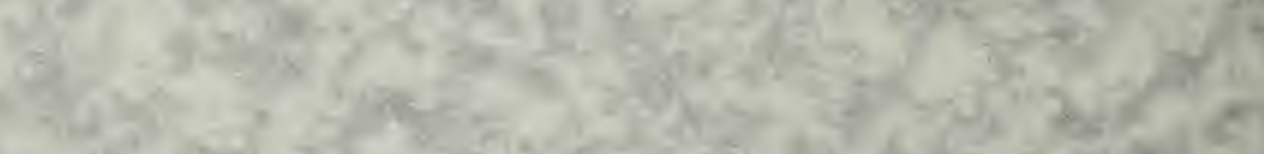

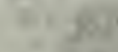

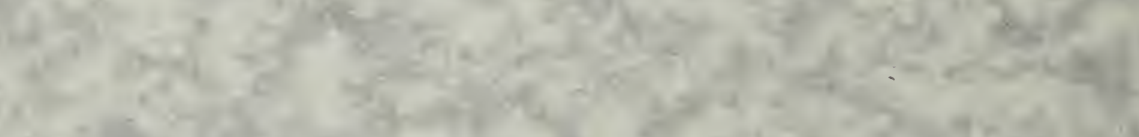

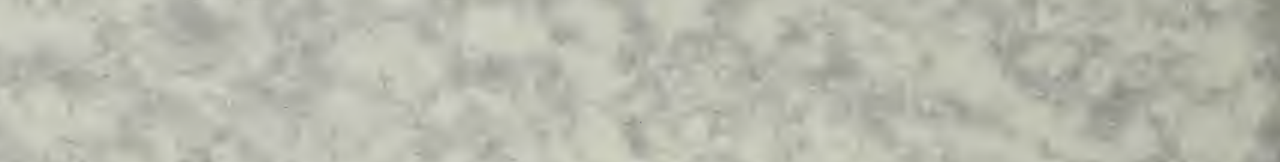

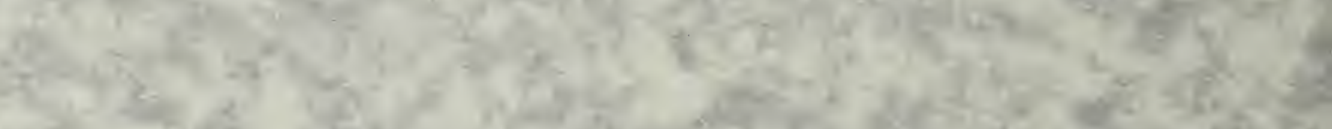
-

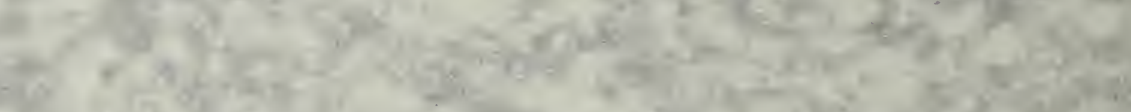

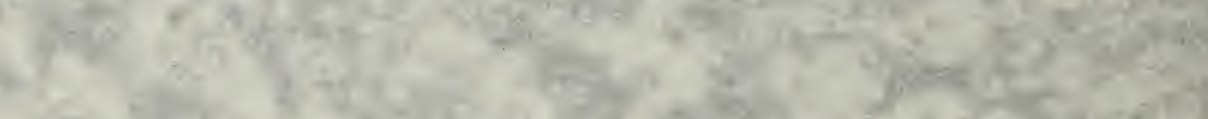

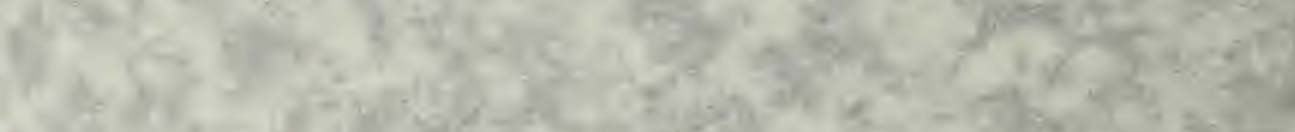

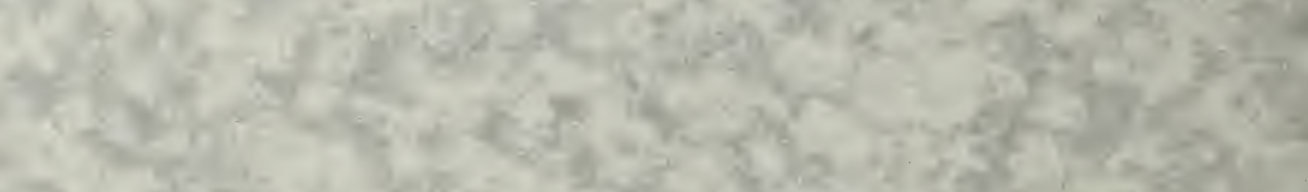

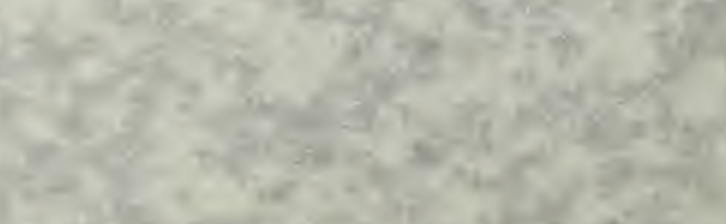

$-2$ 
This Catalog is for Ordering in 1929 Only. If not up to date send for our latest catalog and thus avoid delays.
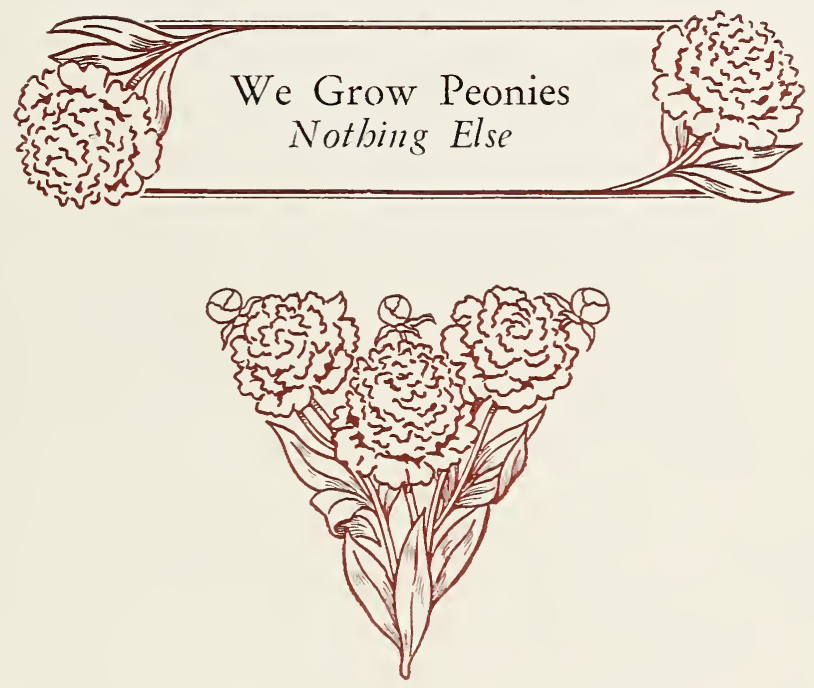

Copjright 1929

by

\section{MOHICAN PEONY GARDENS}

Gardens at

Sinking Spring, Penna.
Office

Reading, Penna.

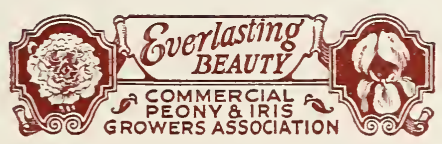




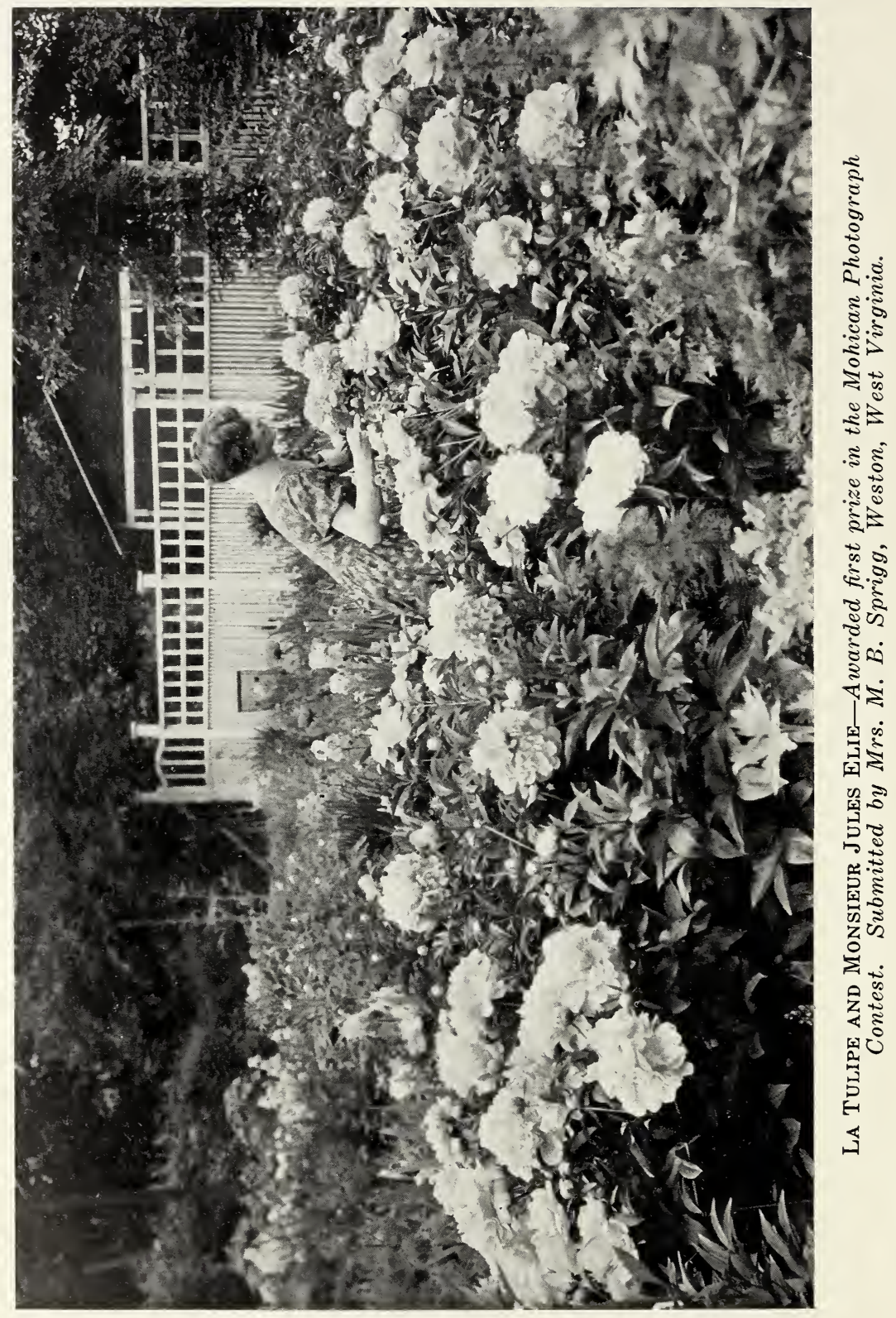




\section{To Mohican Peony Gardens Reading Penna.}

Date

Send to

P.O. Box, R.F.D., or Street Address

Postoffice

Send by

When

Cash enclosed \$

(express or freight)

Shipping dates September first to December first;

Terms and prices as per our 1929 catalog;

No orders filled for less than two dollars;

Shall we substitute in case any varieties should be exhausted?

\begin{tabular}{|c|c|c|c|c|c|}
\hline & & & & & \\
\hline \multirow{2}{*}{ Quantity } & \multirow[b]{2}{*}{ Varieties } & \multicolumn{3}{|c|}{ Price Each } & \multirow{2}{*}{$\begin{array}{l}\text { Total } \\
\text { Amoun }\end{array}$} \\
\hline & & $2-Y_{\text {ear }}$ & $1-$ Year & Divs. & \\
\hline & & & & & \\
\hline & & & & & \\
\hline & & & & & \\
\hline & & & & & \\
\hline & & & & & \\
\hline & & & & & \\
\hline & & & & & \\
\hline & & & & & \\
\hline & & & & & \\
\hline & & & & & \\
\hline & Carried Forward & & & & \\
\hline
\end{tabular}

Should you wish us to send a copy of our catalog to any friends, their names and addresses ma be entered on the back of this order blank. 


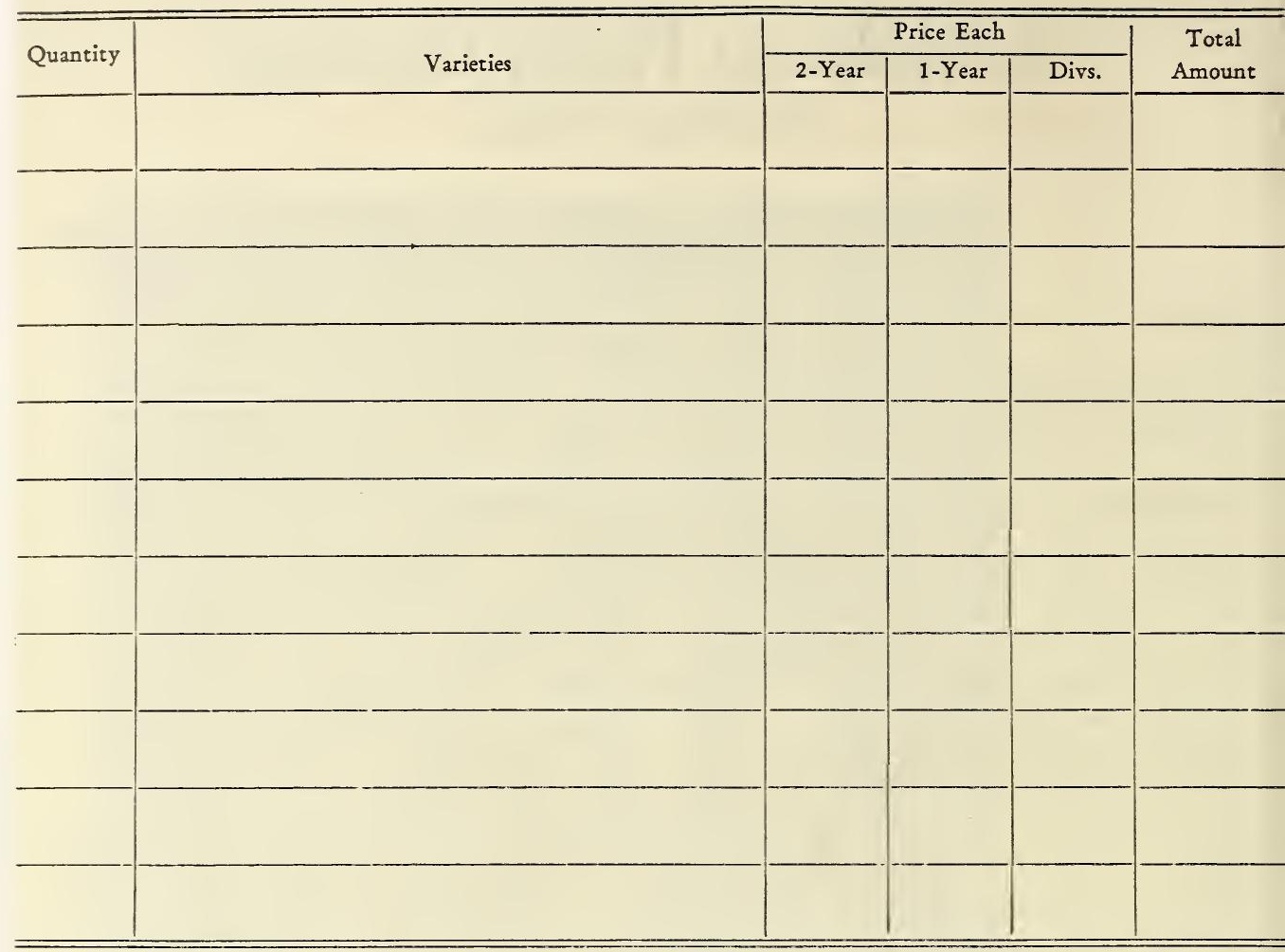

List below the names of friends who might be interested in our catalog. 


\section{Instructions for Ordering}

USE THE ORDER BLANK-Write distinctly in the space provided for all necessary information.

REMITTANCE must be made payable to Mohican Peony Gardens and may be by check, bank draft, cash in registered letter or Express or Post Office Money Order on Reading, $\mathrm{Pa}$. FOREIGN PATRONS must ascertain the correct amount of exchange and add it to their checks, or else remit by Postal Money Order or New York Bank Draft.

DISCOUNT is allowed only when full remittance accompanies order and in no case on the collections offered, according to the following schedule:

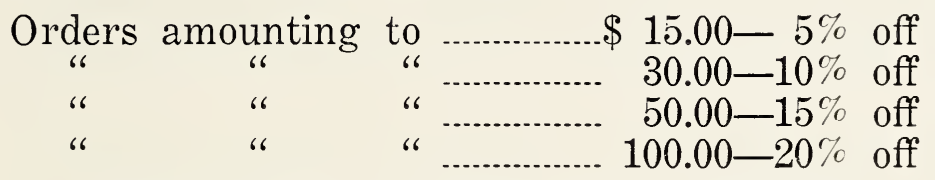

We do not fill orders for less than \$2.00.

TERMS-Open account to those who furnish with order two or more satisfactory credit references. Attention is called to our liberal discounts allowed for remittance with order. (See above).

TRANSPORTATION by express collect or by prepaid parcel post; postage will be billed to customer. West of the Mississippi we recommend freight shipment for economy.

\section{FINANCIAL REFERENCES:}

Sinking Spring Bank, Sinking Spring, Pa. Pennsylvania Trust Company, Reading, Pa.

DO NOT FORGET TO ADD VIGORO TO YOUR ORDER BLANK IF YOU WISH IT INCLUDED

IN YOUR SHIPMENT. 


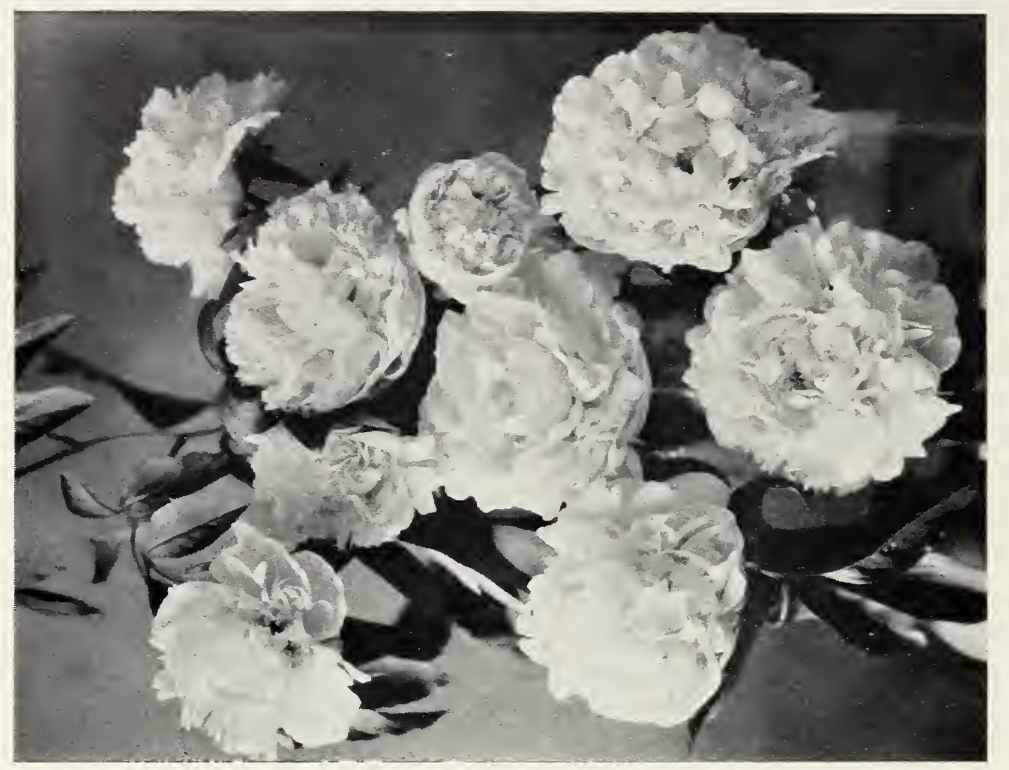

$\frac{2}{2}$

:

$\Xi \Xi$

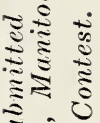

)

ग咅

这

这:

苟

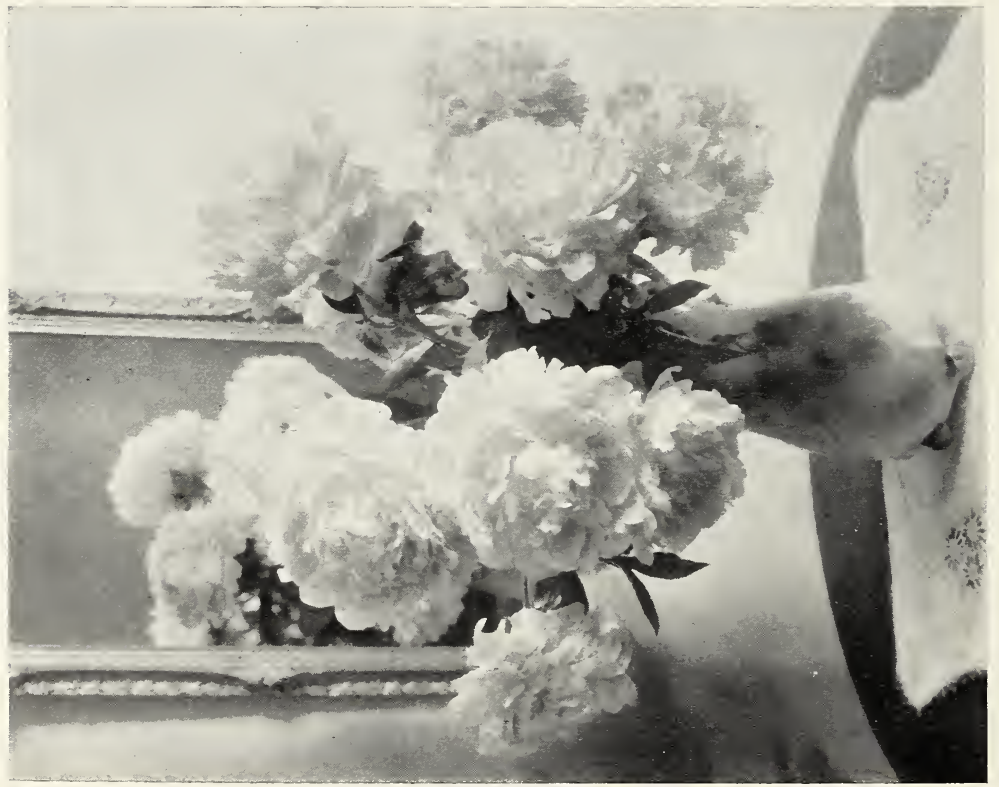

$\stackrel{5}{5}$

$\therefore \quad \approx$

हु

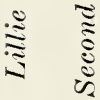

$\stackrel{\infty}{=}$

규

월

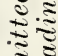

咅

ङ

告

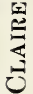




\section{Why Not be a Peony Explorer?}

W ${ }^{\mathrm{E}}$ have been trying to get away from the "stock" descriptions so often used in flower catalogs and to give our readers in a few words a real picture of each variety. Descriptions in this present catalog have for the most part been made up from garden notes jotted down last spring as the different varieties were in bloom, and the Ridgway color notations were arrived at by actual comparison of the flower petal with the Ridgway chart.*

We have tried to restrain our enthusiasm from the too free use of surperlatives and such general adjectives as 'fine', 'enormous', 'beautiful' and so on, but please do not forget that every Peony on our list is a favorite with us and it is hard to stick only to botanical details in introducing them to you.

One result we should like to obtain from our descriptive price list is to tempt your curiosity to order some of the less widely known varieties - we do not mean the new, rare and very expensive sorts, but some of the old French originations, such as Madame Camille Bancel, which are fully as beautifful as their more famous cousins but known only to a few. It has always been a mystery to us why gardeners want only the same varieties that their neighbors are growing and that can be bought anywhere. Is it because only the few are bitten by collector's mania and realize the joy of discovering unfamiliar beauties to introduce to their acquaintance?

May we remind you that every variety on the Mohican list is there because it is beautiful, sturdy and free blooming; we carry no "dead wood". We have honestly tried to give you completely accurate descriptions and when we say, for instance, 'exquisite' we really mean that the particular variety is exquisite-we are not simply using a word to fill out the type line. So, too, when we say "enormous blooms" we mean enormous as compared to the average Peony and not as compared to a daisy. Therefore you run no risk of disappointment in ordering any one of our Peonies whose characteristics interest you, even though you
have never seen it in any one else's garden. Why not be a Peony explorer?

*Ridgway's Color Standards and Nomenclature may be obtained through us or through your book dealer at a price of $\$ 25.00$.

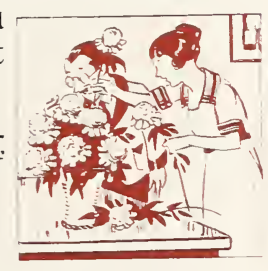

Successful Explorers 


\section{MOHICAN PEONIES M}

\section{The Illustrations}

$\mathrm{T}^{\mathrm{T}}$ should interest those who have not previously received our I catalog to know something about the photographs reproduced in these pages.

The responsive peony urges the photographer, amateur or professional, to do his best to record the beauty it adds to his garden. We encourage this natural impulse by offering more peonies to reward successful pictures.

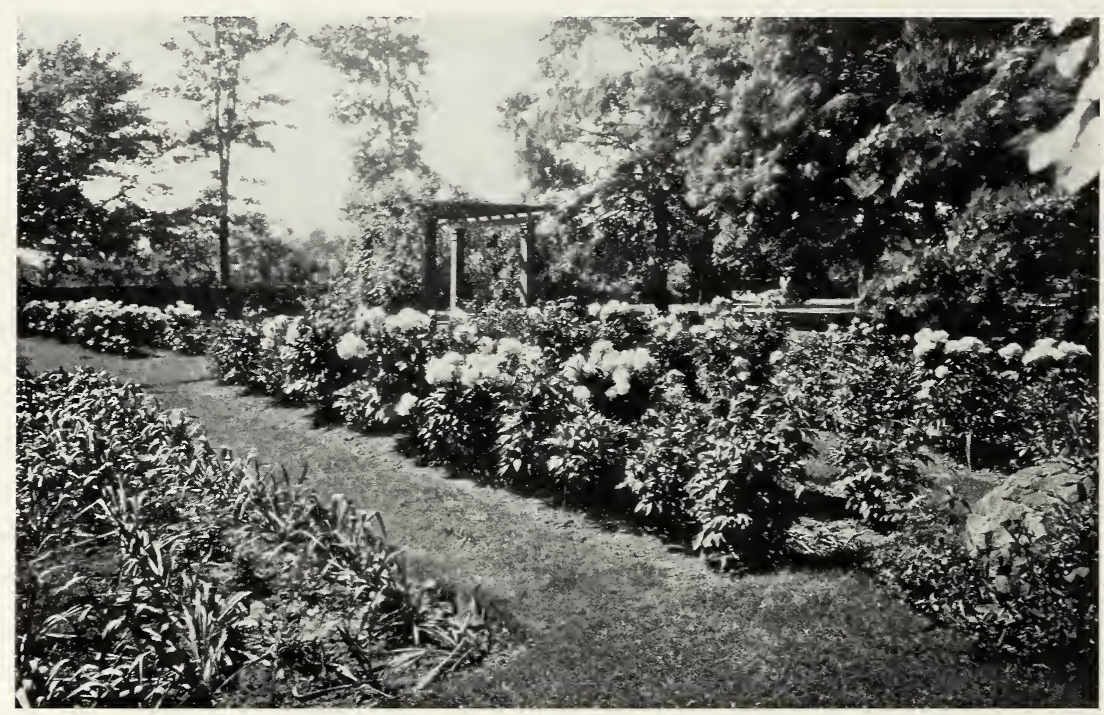

Festiva Maxima AND Therese-Flowers from these plants won first prizes for individual bloom at the Rhode Island Horticultural Society 1928 Show. Submitted by L. W. Jones, Seekonk, Mass. Awarded Third Prize in the Mohican Photograph Contest (See next page).

The Mohican Photograph Contest is intended to promote keener interest in the lovely and often very different characteristics of named varieties. It gives to the gardener a livelier zest for knowing the personalities of his flowers.

The pictures reproduced here are but a few of the many we received last year suggesting the genuine satisfaction of our customers. We believe better testimonials could not be offered. 


\section{MOHICAN PEONIES}

\section{Terms of the Mohican Photograph Contest}

THE prizes offered are Mohican Peonies to be selected, in each case, by the successful contestants.

First Prize: $\$ 50.00$ worth of Mohican Peonies.

Second Prize: $\$ 30.00$ worth of Mohican Peonies.

Third Prize: $\$ 15.00$ worth of Mohican Peonies.

Seven other prizes, each valued at $\$ 5.00$.

Name and address must be plainly written in ink on the back of each photograph; also name of variety of Peony or Peonies shown.

The Peonies photographed must be grown from Mohican roots.

Pictures may be of gardens, plants or cut flowers.

No limit to size of photograph or number submitted by any one person.

Not more than one prize will be allotted to any one contestant.

Photographs must be in our hands by September first.

Any prints submitted may be used in our publications.

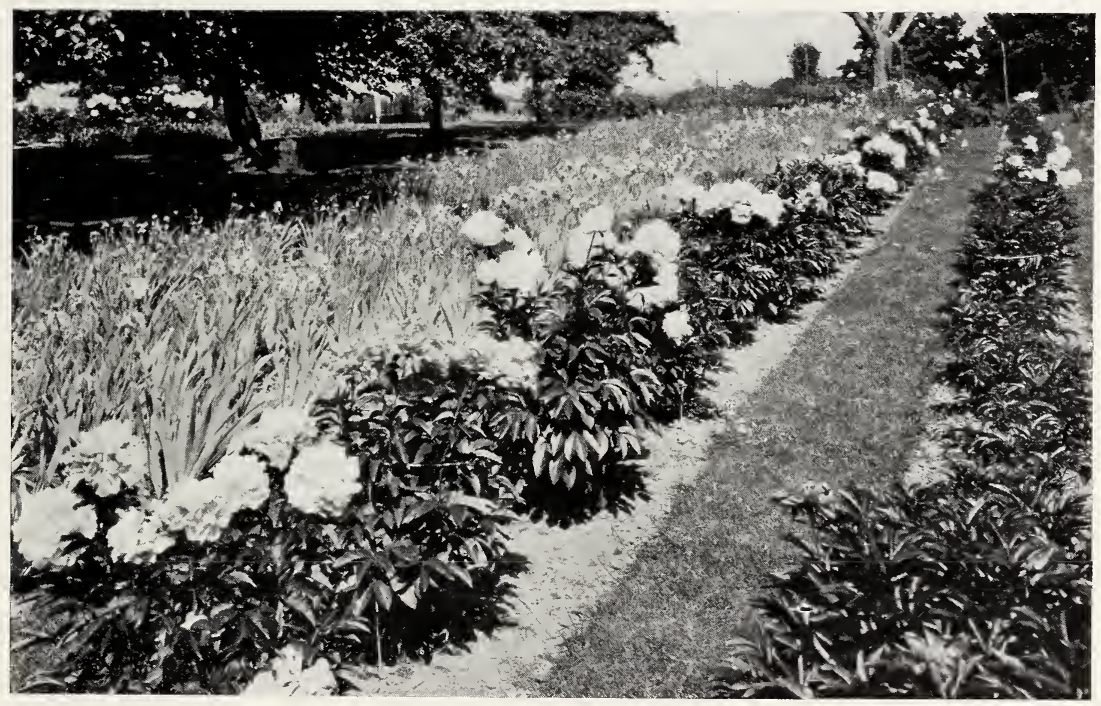

Baroness Schroeder, Felix Crousse and La Rosiere-Submitted by L. W. Jones, Seekonk, Mass. Companion picture to one on opposite page.

All prize-winning pictures will be reproduced with the names and addresses of the successful contestants. Prints will be returned only on request. Prize winning photographs cannot be returned. Send no letterjust the photographs. Address: Mohican Peony Gardens, Reading, Pa. Announcement of awards of prizes will be made in our 1930 catalog. Shipment of the prizes will be made in September by prepaid express. Winners may select the Peonies they most desire and we will do our best to supply just what they may ask for.

Plan Now to Head the List of Winners.

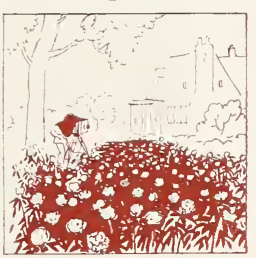




\section{Purchase Your Peonies from True Peony Specialists}

WITH us the Peony is the "whole thing." We grow Peonies and nothing else. We are thus specialists in a sense which possesses legitimate value and significance and the reasonableness of our suggestion, that you purchase your roots from true specialists, becomes apparent.

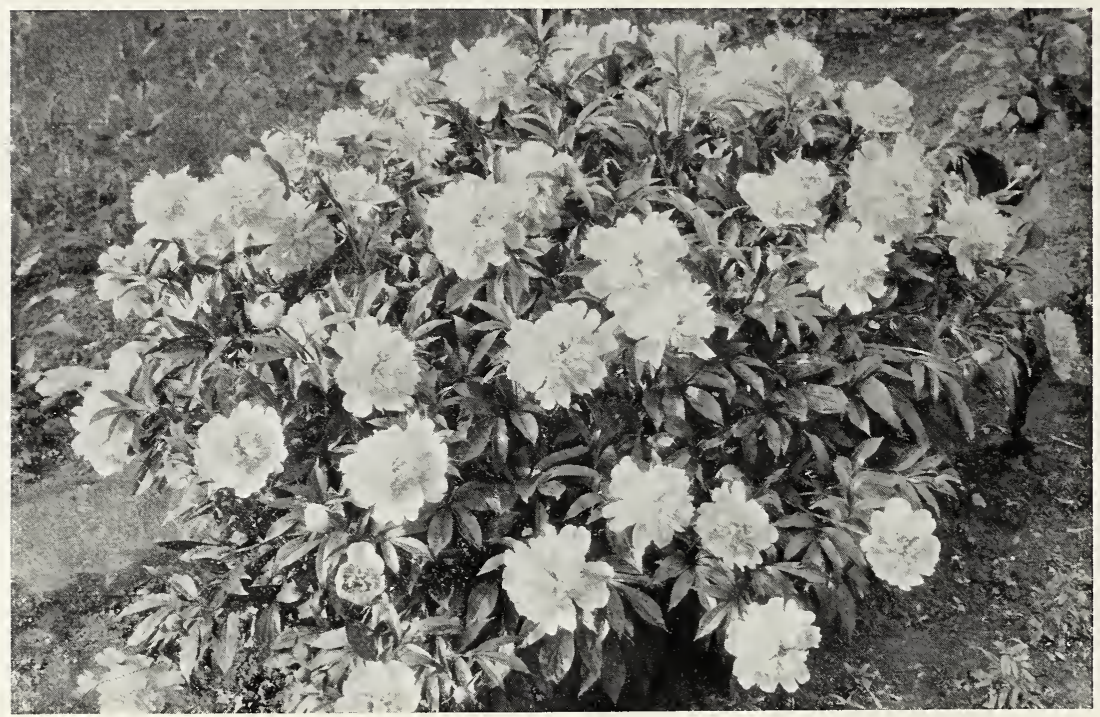

MADAME DUCEL-Three hundred and forty-two buds before disbudding. Submitted by M. Bull, Winnipeg, Canada. Awarded one of Seven Prizes in the Mohican Photograph Contest.

We have labored and studied and spent years of time and a great deal of money to keep our varieties true, and we so guarantee them; that is to say, while we are not infallible and mistakes will occasionally occur, such mistakes are always due to errors in labelling or in shipping and not to mistakes in our varieties. For our fields are thoroughly "rogued" from end to end and we KNOW that there is not a single wrong plant in our entire acreage of Peonies. 


\section{Size and Quality}

The rapidly growing interest in the modern Peony-a popular interest which has attained such proportions that it is not out of harmony to speak of the situation as a "boom in Peonies"brought so prominently to the front the question of size of roots sent out to customers that it is only second in importance to the question of true stock.

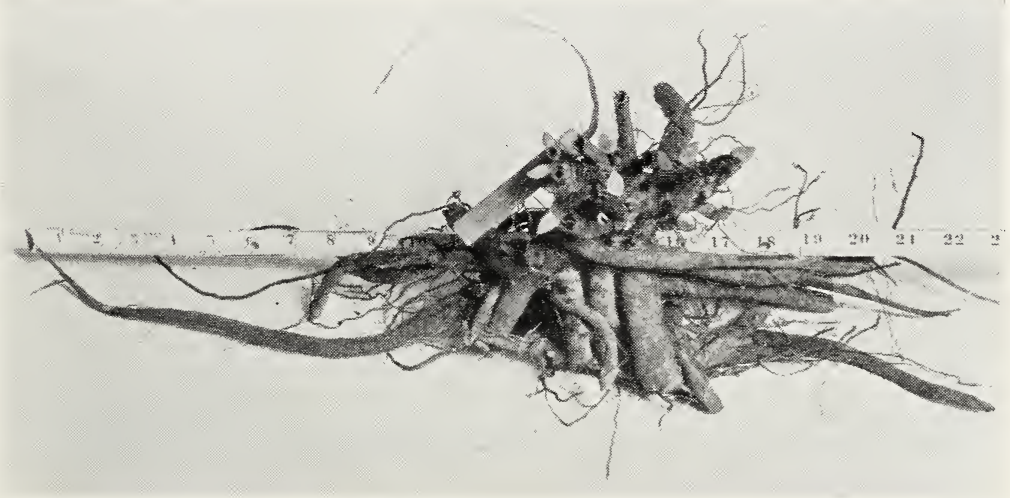

Unlike many growers, we do not take up a more or less mature plant and divide it to the very limit of its endurance. Our divisions are always generous divisions, made with extraordinary care and skill. When our roots are shipped they are in the prime of condition for transplanting.

\section{Our Grade of Stock}

Should you decide to favor us with your valued order, you will, we believe, be surprised at the quality of stock we send you. Our system of growing is such that we are able to send out plants that cannot fail to satisfy the most exacting buyer. The clump we deliver is heavy, vigorous, liberally grown quality stock that will-if properly planted-produce a very satisfactory amount of bloom the first year. All clumps which we ship have bloomed at least once in our gardens and should therefore reasonably be expected to bloom the first season for you.

In soliciting your patronage, therefore, we ask a careful consideration of the points we have made, especially in comparing our prices with those of other growers. We know that our rates, considering the grade of roots we offer, are, to say the least, as low as the same grade of true stock that can be had elsewhere. 


\section{MOHICAN PEONIES}

\section{What Kind of Peonies?}

$\mathrm{T}^{\mathrm{H}}$ HERE are upwards of two thousand varieties of Peonies, and it is a fact -which no grower or nurseryman can honestly dispute-that hundreds of them are so nearly alike as to be practically identical. In any event, only an expert can distinguish the difference. If you send to certain worthy growers for a catalog or list you will be confronted with more or less ac-

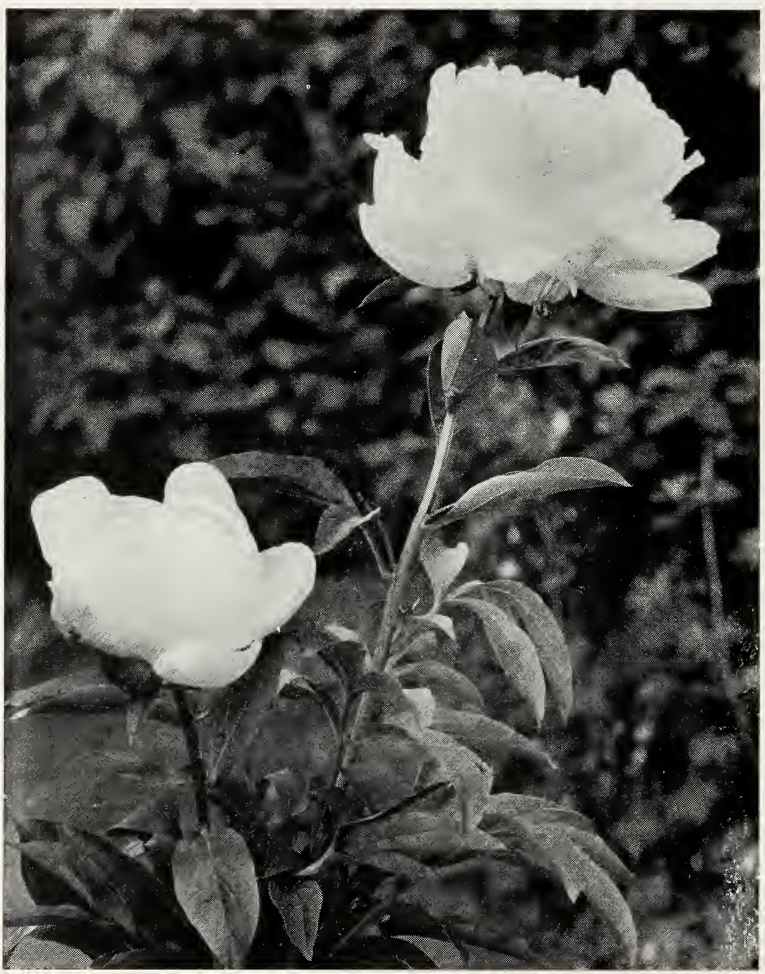

LADY AleXANDRA DUFF-Submitted by Mrs. Frank Pagan, Wellsboro, Penna. Awarded one of Seven Prizes in the Mohican Photograph Contest. curate descriptions of perhaps from two to five hundred sorts. The average retail customer is confused and perplexed by this array. He wants a few of the very best - and that is just the want this catalog will fill.

\section{Our List}

Our list now consists of one hundred carefully selected sorts of Herbaceous Peonies. We are, and have been for years, growing several hundred varieties. The number and character of the varieties in our fields has been constantly changing and shifting as we have cast some out because not entirely worthy and selected new ones as they came into general cultivation. We deem our varieties the choicest and best and the most distinct of the many hundreds in cultivation. The points constantly kept in mind in making our selections have been size, perfection of form, fragrance, vigor of bloom, lasting quality of cut flowers and landscape effect. The entire range of form and color is covered absolutely and all of the exquisite and really worthy new things of

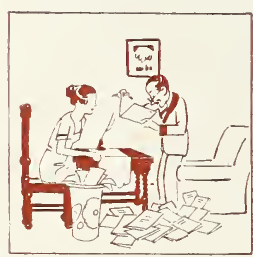
French, British and American production have been added. If you do not find listed in this booklet a variety you think ought to be there, it is because a better one has displaced it or because it is practically identical with one listed. 


\section{Planting}

For permanent planting Peonies should be set from three to four feet apart each way and left undisturbed indefinitely. When it is desired to intersperse Peonies among other hardy garden flowers, or at intervals along a border of shrubs, they should be planted in groups of from three to five, each group to be of one variety. In arranging a bed of Peonies of from fifteen to fifty roots-according to the space at command-we strongly urge the planting of one variety. Although sorts may be had that bloom, in theory,

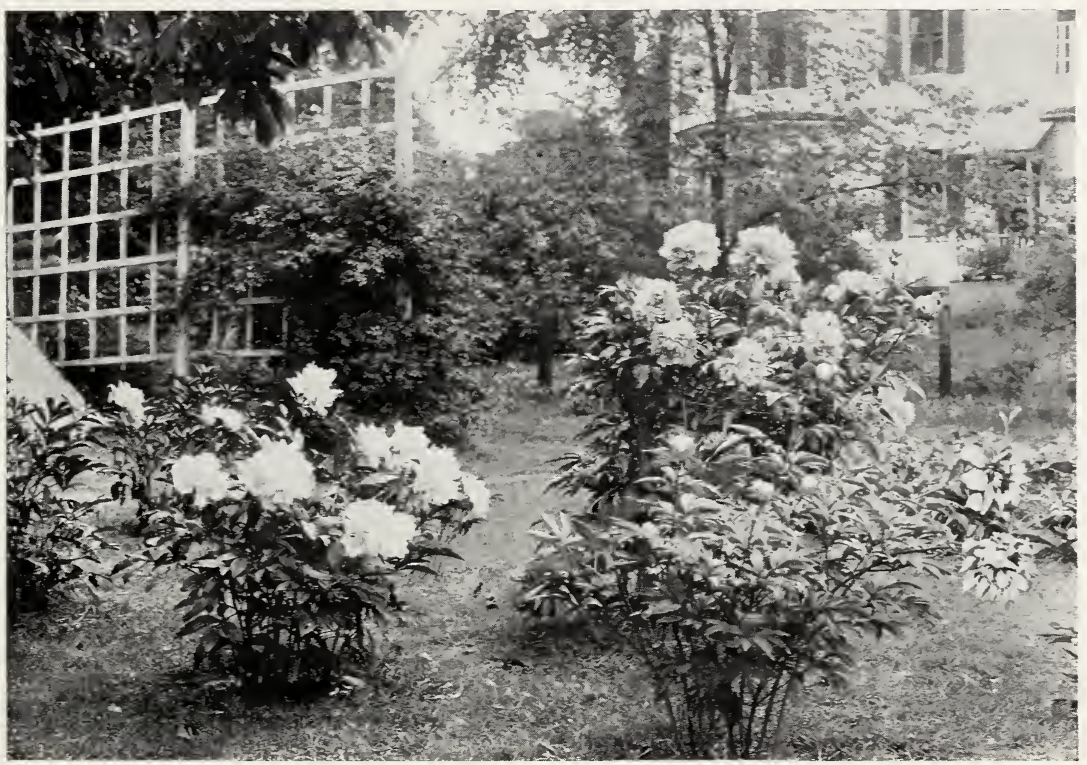

Part of Mohican's Challenge Collection-Submitted by Miss Bessie $\mathrm{N}$. Oviatt, Milford, Conn. Awarded Honorable Mention in the Mohican Photogaph Contest.

about the same time, the result is never satisfactory, for in fact they come straggling into bloom one after the other and the effect desired is never wholly gained. A bed of one fine sort is a magnificient sight.

\section{Planting Season}

The fall season is the only season in which the Peony can successfully be transplanted. To move Peonies at any other season than the months of September, October and November means a setback from which the roots sometimes do not recover for two years. Some general nurserymen will supply Peonies at any season of the year that they can be gotten out of the ground. But when we sell you Peonies we want them to start right in to "work" for you from the beginning, without a setback through moving at the wrong season. We have faith that our methods will thus redound to our credit. We begin digging on September 1st and continue to fill orders as late in the year as the ground remains open. 


\section{Directions for Planting}

Our customers are naturally interested in the proper care of their Peony plants and to meet the need of a reliable guide we have, for many years, sent free with every order our now famous BOOK OF DON'TS which, we

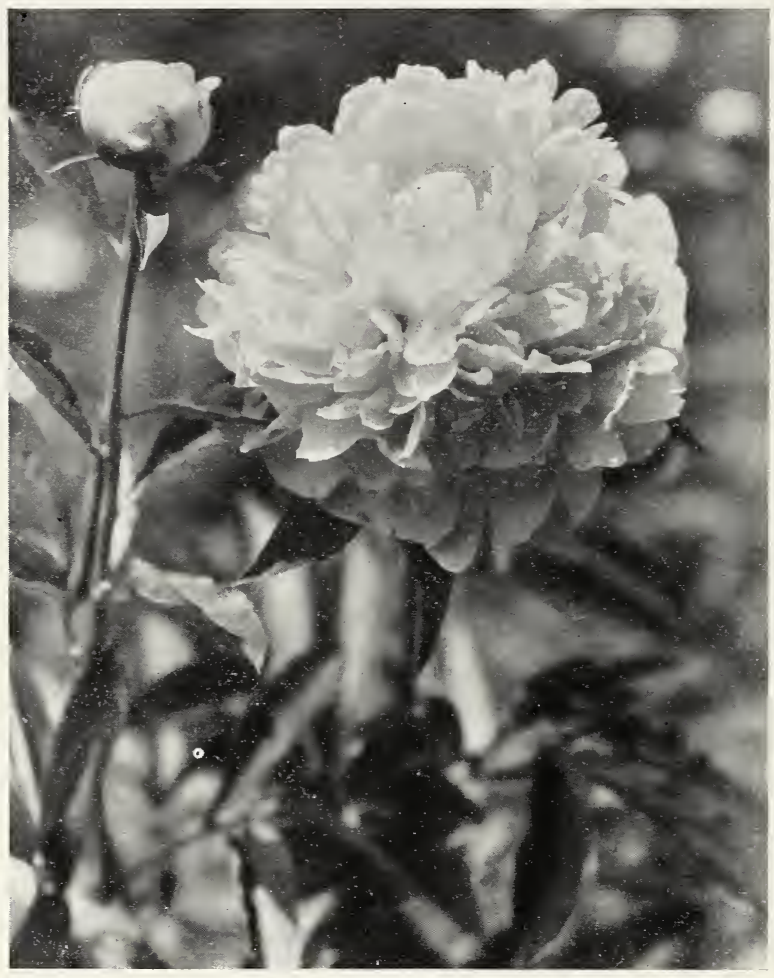

MADAME GeISSLER-As it bloomed the first spring after planting, in the garden of Mrs. Arthur Bodine, Wellsboro, Penna. Awarded one of Seven Prizes in the Mohican Photograph Contest. believe, contains all the advice that is needed to raise Peonies. The few "Don'ts" added to the plant's natural inclination to grow regardless of conditions, makes the Peony a real delight. There are no keen disappointments, no severe losses of labor and love or time and money.

Following the receipt of our Book of Don'ts our customer receives the shipment of well packed, healthy roots. With the "Don'ts" to keep one from errors in planting and with reasonable care on the gardener's part, success is sure to follow.

Except to our customers, the Book of Don'ts is sold at ten cents per copy to cover part of the cost of printing and mailing.

When we revised and i mproved our "Don'ts" in 1925 we recommended as a Peony fertilizer only the use of bone meal and sheep manure, there being at the time no practical commercial fertilizer which we could recommend with confidence. Our Peony roots go to every state in the Union and to several foreign countries and quite naturally, into many different soils. A plant food that will meet all conditions must contain all the elements needed for plant life.

We have at last found a product that we can recommend not only for Peonies but for general use in the garden and on lawns. It is a product of 


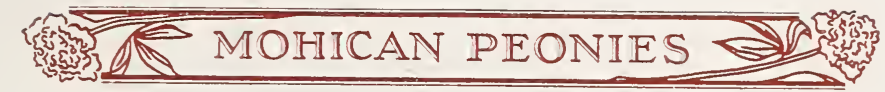

Swift and Company known as Vigoro. Its plant food value is so concentrated that a little goes a long way. It can be purchased, we are informed, in most cities. For the convenience of those who cannot purchase it locally or for those in large cities where the parking problem handicaps local shopping

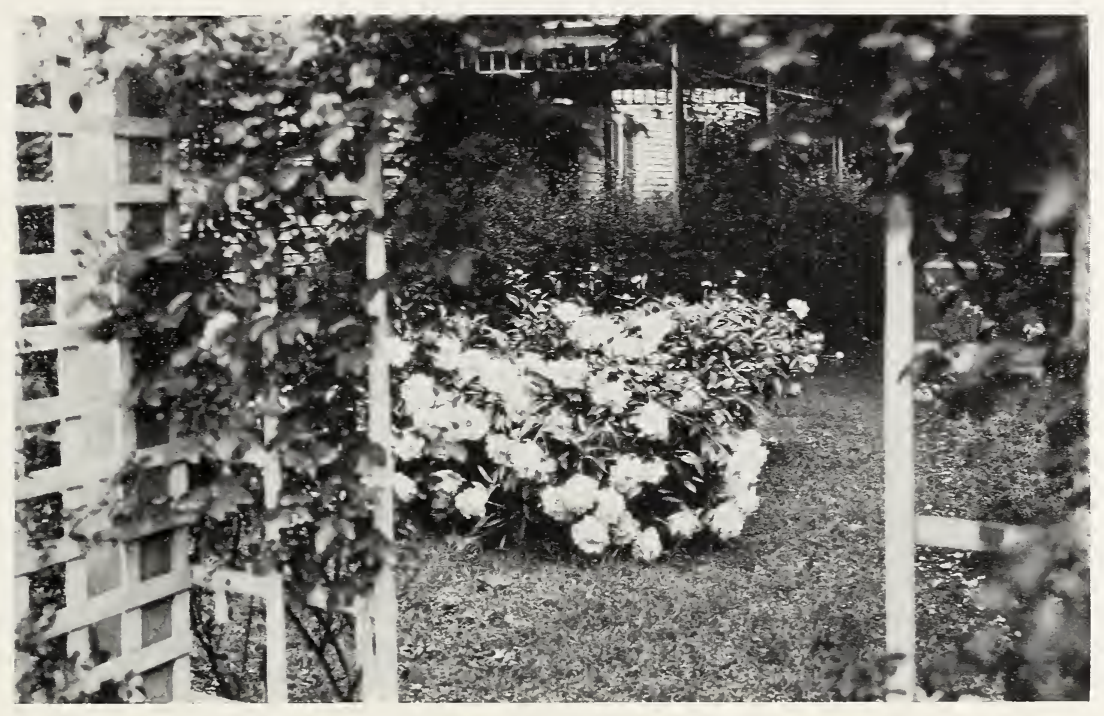

JeANNe D'ARC-Dubmitted by Miss Aura P. Hendrickson, North East, Penna. Awarded one of Seven Prizes in the Mohican Photograph Contest.

we will ship Vigoro with Peony orders. We offer this service with the firm belief that the results will promote the popularity of the "Queen of Flowers" by assuring the successful growth of our hardy stock in the gardens of our customers.

VIGORO. Our desire to have Mohican Peonies prosper in your garden is almost as great as your own ambition for splendid results. Our interest in your garden can be served in no better way than for us to recommend the proper plant food. Vigoro is adaptable to any soil, supplying plants with every element necessary to vigorous growth.

Vigoro ordered is shipped with your Mohican Peony plants. Figure on half a pound per plant, bearing in mind that any surplus can be used to excellent advantage elsewhere in your garden. It is clean, odorless and easy to apply. Directions for use in every package.

100 -lb. bag, $\$ 5.00$ 50-lb. bag, $\$ 3.50$ 25-lb. bag, $\$ 2.00$

5-lb. pkg., $\$ .60$

Page Thirteen

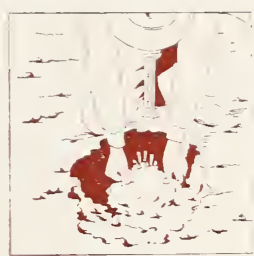

Use Vigoro 


\section{Our Challenge Collection}

This is the group that is always sure to please. We used to think it could not be beaten-now we know it.

This collection may be purchased as a whole at a saving of $25 \%$ or it may be purchased, in two different years, by buying collection No. 2 and collection No. 4, at almost as great a saving.

Or the Challenge Collection may be had in three groups, one group a year if desired, by ordering at different times Collections 3,4 and 5 .

Under no circumstances will we accept orders for our Collections where any changes are requested. Additional varieties should be specified in the space provided on the order blank.

\begin{tabular}{|c|c|c|c|c|c|}
\hline & $\begin{array}{l}\text { Challenge } \\
\text { Collection }\end{array}$ & No. 2 & No. 3 & No. 4 & No. 5 \\
\hline Albert Crousse & $\mathrm{x}$ & $\mathrm{x}$ & ....... & ....... & $\mathrm{x}$ \\
\hline Alexandriana & $\mathbf{x}$ & ....... & ....... & $\mathbf{x}$ & ....... \\
\hline Alice de Julvecourt & $\mathrm{x}$ & $\mathrm{x}$ & $\mathrm{x}$ & ..... & ...... \\
\hline Claire Dubois & $\mathrm{x}$ & ....... & ....... & $\mathrm{x}$ & ....... \\
\hline Couronne d'Or & $\mathrm{x}$ & $\mathrm{x}$ & ....... & $\cdots+.$. & $\mathrm{x}$ \\
\hline Edulis Superba & $\mathrm{x}$ & $\mathrm{x}$ & $\mathbf{x}$ & $\cdots+.$. & -...... \\
\hline Felix Crousse & $\mathrm{x}$ & $\mathrm{x}$ & $\mathbf{x}$ & -..... & $\cdots \cdots$ \\
\hline Festiva Maxima & $\mathbf{x}$ & $\mathrm{x}$ & $\mathrm{x}$ & -..... & -...... \\
\hline Floral Treasure & $\mathrm{x}$ & $\mathrm{x}$ & ....... & ...... & $\mathrm{x}$ \\
\hline \multicolumn{6}{|l|}{ Gloire de Charles } \\
\hline Gombault & $\mathbf{x}$ & $\mathbf{x}$ & $\cdots+.$. & $\cdots \cdots$ & $\mathbf{x}$ \\
\hline Jeanne d'Arc & $\mathrm{x}$ & -..... & $\cdots+.$. & $\mathrm{x}$ & ....... \\
\hline Lafayette & $\mathrm{x}$ & $\mathbf{x}$ & -..... & ...... & $\mathbf{x}$ \\
\hline Livingstone & $\mathrm{x}$ & ...... & ...... & $\mathrm{x}$ & ...... \\
\hline Mme. Bucquet & $\mathrm{x}$ & $\cdots \cdots$ & -..... & $\mathrm{x}$ & ...... \\
\hline Mme. de Verneville & $\mathrm{x}$ & $\mathrm{x}$ & $\mathrm{x}$ & ...... & ...... \\
\hline Mme. Ducel & $\mathrm{x}$ & $\cdots . .$. & -..... & $\mathrm{x}$ & $\ldots . .$. \\
\hline Mme. Emile Galle & $\mathbf{x}$ & $\mathrm{x}$ & $\mathrm{x}$ & & -..... \\
\hline Mme. Geissler & $\mathrm{x}$ & ..... & $\cdots+.$. & $\mathrm{x}$ & ....... \\
\hline Marie Lemoine & $\mathrm{x}$ & $\mathrm{x}$ & $\mathrm{x}$ & -..... & ...... \\
\hline Modeste Guerin & $\mathrm{x}$ & $\mathrm{x}$ & $\mathrm{x}$ & ...... & ...... \\
\hline M. Dupont & $\mathrm{x}$ & ...... & ...... & $\mathrm{x}$ & ....... \\
\hline M. Jules Elie & $\mathrm{x}$ & $\mathrm{x}$ & -..... & ..... & $\mathbf{x}$ \\
\hline $\begin{array}{l}\text { Sarah Bernhardt } \\
\text { Souvenir de l'Ex }\end{array}$ & $x$ & $\cdots \cdots$ & -..... & $\mathrm{x}$ & -..... \\
\hline \multirow{2}{*}{\multicolumn{6}{|c|}{$\begin{array}{l}\text { de Bordeaux } \\
\text { Triomphe de l'Ex }\end{array}$}} \\
\hline & $\mathrm{x}$ & $\mathrm{x}$ & ........ & ........ & $\mathrm{x}$ \\
\hline & & & & & \\
\hline
\end{tabular}

\section{Collection price on}

$\begin{array}{rrrrrr}\text { one year roots....\$26.75 } & \$ 16.10 & \$ 9.00 & \$ 12.00 & \$ 7.50 \\ \text {.on divisions... } 21.00 & 12.90 & 7.20 & 9.60 & 6.00\end{array}$




\section{Special Collections}

If you could walk with me through fields of Peonies in bloom and point out those you like the best, ordering them on the spot as do visitors to our gardens, you would have blooming in your garden next spring the seven popular Peonies which we call VISITORS' CHOICE :

Albert Crousse, late, light pink.

Felix Crousse, late mid-season, red.

Festiva Maxima, very early, white.

Madame Geissler, late mid-season, deep pink.

Marie Lemoine, late, ivory white.

Souvenir de l'Exposition de Bordeaux, mid-season, red.

Triomphe de l'Exposition de Lille, mid-season, light pink.

Collection Price on One Year Roots \$6.25; On Divisions \$5.00.

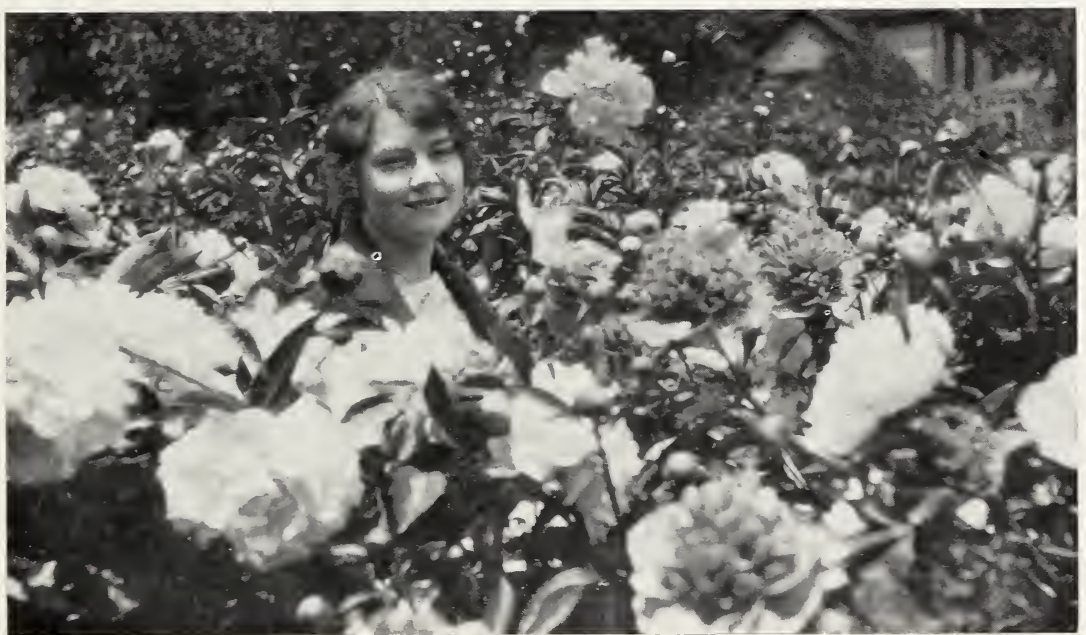

A Choice Collection-Submitted by A. J. Parks, Syracuse, N. Y. Awarded one of Seven Prizes in the Mohican Photograph Contest.

Anyone desiring a touch of glorious red, bordered with rose and white, cannot make a mistake in selecting the well-balanced group which is called

THE PENTAGON :

Avalanche, mid-season, white.

Eugene Reignoux, early mid-season, deep pink.

Felix Crousse, late mid-season, red.

Madame Calot, early, light pink.

Marie Lemoine, late, ivory white.

Collection Price on One Year Roots $\$ 4.80 ; \quad$ On Divisions $\$ 3.85$. 


\section{MOHICAN PEONIES}

THE DIXIE COLLECTION :

The following collection is composed only of varieties which have been proven successful Southerners. Many other varieties listed in our catalog were found flourishing in southern gardens, but we have chosen a rep-

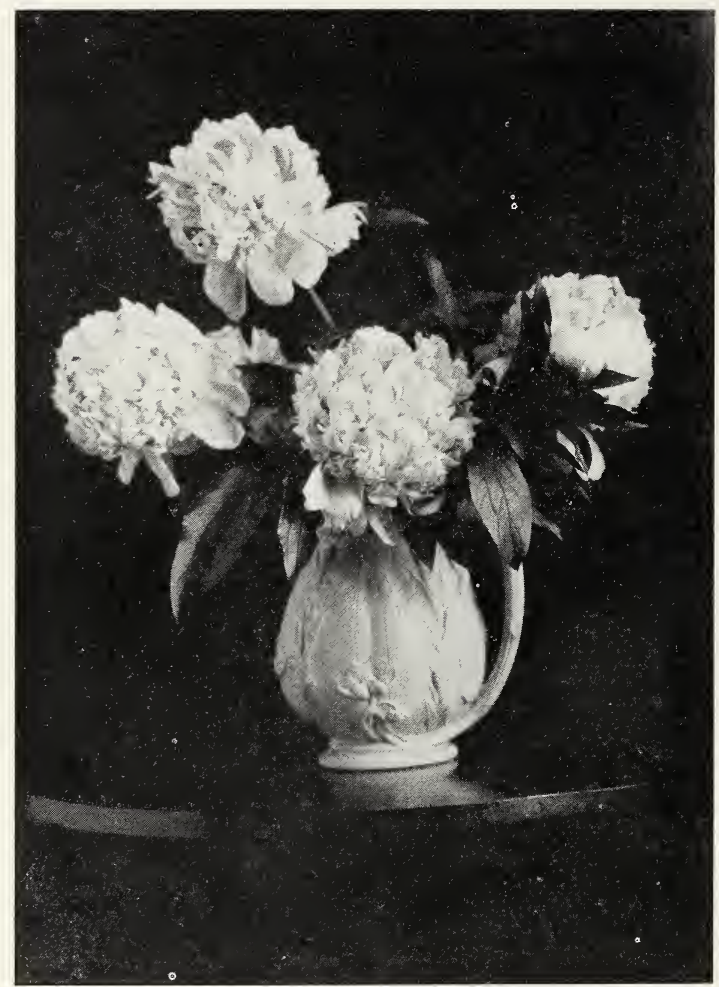

Monsieur Jules Elie-Submitted by Mrs. A. L. Fowlkes, South Boston, Virginia. Awarded Honorable Mention in the Mohican Photograph Contest. resentative group that covers the entire color and season range. All of these "Dixies" are fragrant.

Baroness Schroeder

Edulis Superba

Festiva Maxima Madarne Bucquet Madame Calot Monsieur Jules Elie Petite Renee

\section{Collection Price on One} Year Roots \$10.00; On Divisions \$8.00.

In offering the foregoing collection we wish to warn you that for southern planting a moisture-retaining soil is absolutely necessary, preferably heavy loam. If your soil is sandy, a clay subsoil is very important.

\section{THREE PERFECT} PINKS:

It would be difficult to select from any garden of any flowers three more beautiful pinks than our little group of

La Perle

Mme. Auguste Dessert Therese

Collection Price on One Year Roots \$6.25;

On Divisions \$5.00

THE SEXTET FROM MOHICAN :

The harmony of a sextet of beauty is always inspiring and if you seek a wealth of color you will be delighted with

Aurore

Coquelin

La Tendresse,

La Tulipe,

M. Boucharlat Aine,

Souvenir de l'Exposition de Bordeaux,

Collection Price on One Year Roots \$5.20; On Divisions \$4.15. 


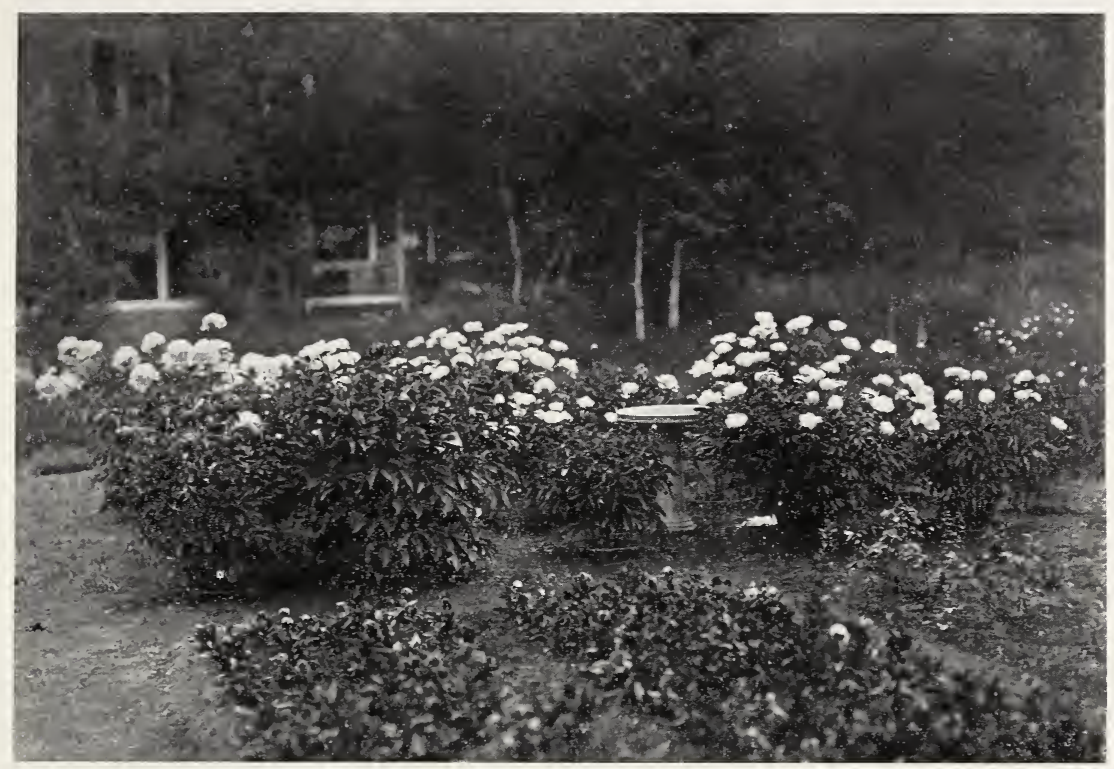

Mohican's Collection “Three Perfect Pinks"-Submitted by Mrs. Herbert L. Welch, Haines Landing, Maine.

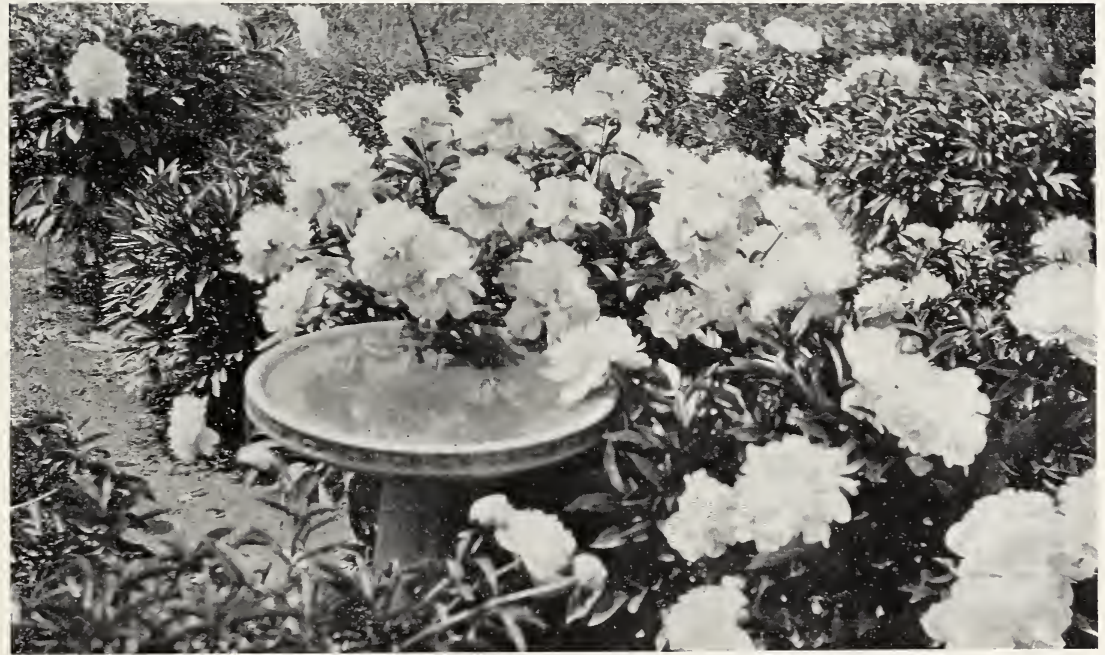

A Close Up of "Three Perfect Pinks"-Submitted by Mrs. Herbert L. Welch, Haines Landing, Maine.

Awarded one of Seven Prizes in the Mohican Photograph Contest. 


\section{Descriptive Chart}

\begin{tabular}{|c|c|c|c|c|c|c|c|}
\hline Color & $\begin{array}{c}\text { Fra- } \\
\text { grance }\end{array}$ & Season & Variety & Form & Vigor & Size & $\begin{array}{l}\text { For } \\
\text { Cut- } \\
\text { ting }\end{array}$ \\
\hline Deep Red & & Early & Adolphe Rousseau & Semi-double & $\mathrm{X}$ & $\mathrm{XX}$ & \\
\hline Light Pink & $\mathrm{X}$ & Late & Albert Crousse & Bomb & $\mathrm{XX}$ & $\mathrm{xx}$ & $\mathrm{X}$ \\
\hline Light Pink & $\mathrm{X}$ & Early & Alexandriana & Double & $\mathrm{XX}$ & $\mathrm{XX}$ & $\mathrm{X}$ \\
\hline Tri-color & $\mathrm{X}$ & Early-mid. & Alice de Julvecourt & Crown & $\mathrm{XX}$ & $\mathrm{x}$ & $\mathrm{X}$ \\
\hline Cream-white & $\ldots$ & Late-mid. & Alsace Lorraine & Semi-double & $\mathrm{X}$ & $\mathrm{XX}$ & . \\
\hline Light Pink & $\mathrm{X}$ & Midseason & Asa Gray & Semi-double & $\mathrm{X}$ & $\mathrm{X}$ & $\mathrm{XX}$ \\
\hline Tri-color & $\mathrm{X}$ & Midseason & Aurore & Semi-double & $\mathrm{X}$ & $\mathrm{X}$ & $\mathrm{XX}$ \\
\hline White & $\mathrm{X}$ & Midseason & Avalanche & Crown & $\mathrm{XX}$ & $\mathrm{xx}$ & $\mathrm{XX}$ \\
\hline Light Pink & $\ldots$ & Very early & Banksi & Tree & $\mathrm{XX}$ & $\mathrm{xX}$ & $\cdots$ \\
\hline White & $\mathrm{X}$ & Midseason & Baroness Schroeder & Double & $\mathrm{XX}$ & $\mathrm{XX}$ & $\mathrm{xX}$ \\
\hline White & $\mathrm{X}$ & Early-mid. & Boule de Neige & Semi-double & $\mathrm{XX}$ & $\mathrm{X}$ & $\mathrm{XX}$ \\
\hline Red & $\mathrm{XX}$ & Late-mid. & Charles Verdier & Double & $\mathrm{XX}$ & $\mathrm{X}$ & $\cdots$ \\
\hline Red & $\ldots$ & Very early & Cherry Hill & Double & $\mathrm{X}$ & $\mathrm{X}$ & $\mathrm{XX}$ \\
\hline Deep Pink & $\mathrm{X}$ & Midseason & Claire Dubois & Double & $\mathrm{XX}$ & $\mathrm{XX}$ & $\mathrm{X}$ \\
\hline Pale Pink & $\ldots$ & Midseason & Clio & Single & $\mathrm{XX}$ & $\mathrm{X}$ & \\
\hline Red & $\mathrm{x}$ & Late & Constant Devred & Double & $\mathrm{XX}$ & $\mathrm{XX}$ & $\mathrm{X}$ \\
\hline $\begin{array}{l}\text { Deep Pink } \\
\text { Yellow }\end{array}$ & $\cdots$ & Very early & Coquelin & Semi-double & $\mathrm{X}$ & $\mathrm{x}$ & \\
\hline \& White & $\mathrm{XX}$ & Late & Couronne d'Or & Semi-double & $\mathrm{XX}$ & $\mathrm{XX}$ & $\mathrm{XX}$ \\
\hline $\begin{array}{l}\text { Light Pink } \\
\text { Yellow }\end{array}$ & $\mathrm{X}$ & Very late & Dorchester & Double & $\mathrm{xx}$ & $\mathrm{XX}$ & $\mathrm{X}$ \\
\hline \& White & $\mathrm{X}$ & Early & Duchesse de Nemours & Crown & $\mathrm{xX}$ & $\mathrm{X}$ & $\mathrm{XX}$ \\
\hline White & $\mathrm{X}$ & Late-mid & Duke of Wellington & Bomb & $\mathrm{XX}$ & $\mathrm{XX}$ & $\mathrm{xX}$ \\
\hline Deep Pink & $\mathrm{XX}$ & Very early & Edulis Superba & Crown & $\mathrm{XX}$ & $\mathrm{XX}$ & $\mathrm{xx}$ \\
\hline Light Pink & $\mathrm{xX}$ & Midseason & Edwin C. Shaw & Double & $\mathrm{X}$ & $\mathrm{X}$ & $\mathrm{xx}$ \\
\hline Red & $\cdots$ & Late-mid. & Emperor Nicholas & Bomb & $\mathrm{X}$ & $\mathrm{X}$ & $\cdots$ \\
\hline Deep Pink & $\cdots$ & Early-mid. & Eugene Reignoux & Semi-double & $\mathrm{X}$ & $\mathrm{XX}$ & ... \\
\hline Light Pink & $\mathrm{xx}$ & Late & Eugene Verdier & Double & $\mathrm{XX}$ & $\mathrm{XX}$ & $\cdots$ \\
\hline Light Pink & $\mathrm{X}$ & Midseason & Eugenie Verdier & Semi-double & $\mathrm{x}$ & $\mathrm{XX}$ & $\mathrm{XX}$ \\
\hline Red & $\mathrm{X}$ & Midseason & Felix Crousse & Bomb & $\mathrm{XX}$ & $\mathrm{XX}$ & $\mathrm{XX}$ \\
\hline White & $\mathrm{X}$ & Very early & Festiva Maxima & Double & $\mathrm{XX}$ & $\mathrm{xX}$ & $\mathrm{XX}$ \\
\hline Light Pink & $\mathrm{X}$ & Midseason & Floral Treasure & Double & $\mathrm{XX}$ & $\mathrm{X}$ & $\mathrm{XX}$ \\
\hline Light Pink & $\mathrm{X}$ & Midseason & Germaine Bigot & Crown & $\mathrm{x}$ & $\mathrm{XX}$ & $\mathrm{X}$ \\
\hline Tri-color & $\mathrm{X}$ & Midseason & Gloire de C. Gombault & Crown & $\mathrm{XX}$ & $\mathrm{XX}$ & $\mathrm{xX}$ \\
\hline Light Pink & $\mathrm{XX}$ & Very late & Grandiflora & Double & $\mathrm{XX}$ & $\mathrm{XX}$ & $\mathrm{XX}$ \\
\hline White & $\mathrm{X}$ & Early & Grand. Nivea Plena & Double & $\mathrm{X}$ & $\mathrm{X}$ & $\mathrm{X}$ \\
\hline Tri-color & $\mathrm{X}$ & Midseason & Jeanne d'Arc & Crown & $\mathrm{XX}$ & $\mathrm{X}$ & $\cdots$ \\
\hline Light Pink & $\mathrm{x}$ & Early & Judge Berry & Semi-double & $\mathrm{X}$ & $\mathrm{xX}$ & . \\
\hline Red & $\cdots$ & Midseason & Karl Rosefield & Semi-double & $\mathrm{XX}$ & $\mathrm{XX}$ & XX \\
\hline White & $\mathrm{X}$ & Early-mid. & Kelway's Glorious & Double & $\mathrm{XX}$ & $\mathrm{XX}$ & $\mathrm{xx}$ \\
\hline Red & $\mathrm{X}$ & Early-mid. & King of England & Japanese & $\mathrm{X}$ & $\mathrm{XX}$ & $\mathrm{X}$ \\
\hline Pale Pink & $\mathrm{XX}$ & Early & Lady Alexandra Duff & Semi-double & $\mathrm{XX}$ & $\mathrm{XX}$ & $\mathrm{x}$ \\
\hline Deep Pink & $\mathrm{XX}$ & Midseason & Lafayette & Double & $\mathrm{X}$ & $\mathrm{XX}$ & $\mathrm{XX}$ \\
\hline Light Pink & $\mathrm{XX}$ & Midseason & Lamartine & Double & $\mathrm{X}$ & $\mathrm{X}$ & $\mathrm{X}$ \\
\hline Light Pink & $\mathrm{X}$ & Late-mid & La Perle & Double & $\mathrm{X}$ & $\mathrm{X}$ & $\mathrm{XX}$ \\
\hline Cream-white & $\mathrm{XX}$ & Midseason & La Rosiere & Semi-double & $\mathrm{X}$ & $\mathrm{XX}$ & $\mathrm{xX}$ \\
\hline White & $\mathrm{X}$ & Early-mid. & La Tendresse & Double & $\mathrm{XX}$ & $\mathrm{XX}$ & $\mathrm{X}$ \\
\hline Light Pink & $\mathrm{X}$ & Late-mid. & La Tulipe & Semi-double & $\mathrm{X}$ & $\mathrm{X}$ & $\mathrm{X}$ \\
\hline Yellow & $\mathrm{X}$ & Early-mid. & Laura Dessert & Anemone & $\mathrm{X}$ & $\mathrm{X}$ & $\mathrm{X}$ \\
\hline White & $\mathrm{XX}$ & Midseason & Le Cygne & Double & $\mathrm{XX}$ & $\mathrm{XX}$ & $\mathrm{XX}$ \\
\hline Light Pink & $\cdots$ & Very late & Livingstone & Double & $\mathrm{xx}$ & $\mathrm{XX}$ & $\mathrm{X}$ \\
\hline Red & $\dddot{x}$ & Midseason & Longfellow & Semi-double & $\mathrm{X}$ & $\mathrm{X}$ & $\mathrm{XX}$ \\
\hline Light Pink & $\mathrm{X}$ & Early-mid. & Mme. Auguste Dessert & Semi-double & $\mathrm{XX}$ & $\mathrm{XX}$ & $\mathrm{X}$ \\
\hline Deep Red & $\mathrm{X}$ & Midseason & Mme. Bucquet & Semi-double & $\mathrm{X}$ & $\mathrm{X}$ & $\mathrm{X}$ \\
\hline
\end{tabular}

See pages 21 to 40 for detailed descriptions and prices. 


\section{Descriptive Chart}

\begin{tabular}{|c|c|c|c|c|c|c|c|}
\hline Color & $\begin{array}{c}\text { Fra- } \\
\text { grance }\end{array}$ & Seascn & Variety & Form & Vigor & Size & $\begin{array}{l}\text { For } \\
\text { Cut- } \\
\text { ting }\end{array}$ \\
\hline Pale Pink & $\mathrm{x}$ & Early-mic. & Mme. Calot & Double & $\mathrm{xx}$ & $\mathrm{xx}$ & $\mathrm{xx}$ \\
\hline Light Pink & $\mathrm{x}$ & Late & Mme. Camille Bancel & Double & $\mathrm{XX}$ & $\mathrm{X}$ & $\mathrm{X}$ \\
\hline White & $\mathrm{x}$ & Late-mid. & Mme. Crousse & Crown & $\mathrm{xx}$ & $\mathrm{xx}$ & $\mathrm{xx}$ \\
\hline Light Pink & $\mathrm{X}$ & Late & Mme. de Galhau & Double & $\mathrm{XX}$ & $\mathrm{X}$ & $\mathrm{x}$ \\
\hline Tri-color & $\mathrm{xX}$ & Midseason & Mme. de Vatry & Crown & $\mathrm{X}$ & $\mathrm{x}$ & $\mathrm{xx}$ \\
\hline White & $\mathrm{Xx}$ & Early & Mme. de Verneville & Bomb & $\mathrm{XX}$ & $\mathrm{xx}$ & $\mathrm{Xx}$ \\
\hline Deep Pink & $\mathrm{X}$ & Early-mid. & Mme. Ducel & Bomb & $\mathrm{XX}$ & $\mathrm{XX}$ & $\mathrm{xx}$ \\
\hline Light Pink & $\mathrm{Xx}$ & Late-mid. & Mme. Emile Galle & Double & $\mathrm{xx}$ & $\mathrm{X}$ & $\mathrm{x}$ \\
\hline White & $\mathrm{xX}$ & Midseason & Mme. Emile Lemoine & Semi-double & $\mathrm{xx}$ & $\mathrm{Xx}$ & $\mathrm{xx}$ \\
\hline Deep Pink & $\mathrm{X}$ & Late-mid. & Mme. Forel & Double & $\mathrm{x}$ & $\mathrm{xx}$ & $\ldots$ \\
\hline Deep Pink & $\mathrm{X}$ & Late-mid. & Mme. Geissler & Double & $\mathrm{XX}$ & $\mathrm{xx}$ & $\mathrm{xx}$ \\
\hline Light Pink & $\mathrm{X}$ & Midseason & Mlle. Rousseau & Semi-double & $\mathrm{xx}$ & $\mathrm{xx}$ & $\mathrm{x}$ \\
\hline White & $\mathrm{Xx}$ & Midseaso! & Marcelle Dessert & Crown & $\mathrm{X}$ & $\mathrm{xx}$ & $\mathrm{X}$ \\
\hline Light Pink & $\mathrm{x}$ & Late-mic. & Marguerite Gerard & Semi-double & $\mathrm{xx}$ & $\mathrm{xx}$ & $\mathrm{X}$ \\
\hline Light Pink & $\mathrm{X}$ & Midseason & Marie Crousse & Bomb & $\mathrm{XX}$ & $\mathrm{xx}$ & $\mathrm{x}$ \\
\hline White & $\mathrm{XX}$ & Midseason & Marie Jacquin & Single & $\mathrm{X}$ & $\mathrm{x}$ & $\ldots$ \\
\hline Ivory White & $\mathrm{X}$ & Late & Marie Lemoine & Double & $\mathrm{xx}$ & $\mathrm{Xx}$ & $\mathrm{xx}$ \\
\hline Deep Pink & $\mathrm{xx}$ & Late & Martha Bullock & Double & $\mathrm{xx}$ & $\mathrm{xX}$ & $x$ \\
\hline Red & $\cdots$ & Midseason & Mary Brand & Semi-double & $\mathrm{x}$ & $\mathrm{x}$ & $\mathrm{XX}$ \\
\hline Light Pink & $\mathrm{xx}$ & Late & Mathilde de Roseneck & Double & $\mathrm{xx}$ & $x x$ & $\mathrm{X}$ \\
\hline Red & $\ldots$ & Midseason & Mikado & Japanese & $\mathrm{x}$ & $\mathrm{X}$ & $\mathrm{X}$ \\
\hline Pale Pink & .. & Late & Milton Hill & Double & $\mathrm{Xx}$ & $\mathrm{xx}$ & $\mathrm{xx}$ \\
\hline Light Pink & $\mathrm{X}$ & Late & Modele de Perfection & Double & $\mathrm{X}$ & $\mathrm{X}$ & $\mathrm{x}$ \\
\hline Deep Pink & $\mathrm{X}$ & Midseason & Modeste Guerin & Bomb & $\mathrm{xX}$ & $\mathrm{xx}$ & $\mathrm{x}$ \\
\hline Light Pink & $\mathrm{X}$ & Late-mid. & M. Boucharlat Aine & Semi-double & $\mathrm{XX}$ & $\mathrm{xx}$ & $\cdots$ \\
\hline Ivory White & $\mathrm{X}$ & Late-mid. & M. Dupont & Semi-double & $\mathrm{XX}$ & $\mathrm{xx}$ & $\mathrm{Xx}$ \\
\hline Deep Pink & $\mathrm{xx}$ & Early & M. Jules Elie & Bomb & $\mathrm{XX}$ & $\mathrm{XX}$ & $\mathrm{xx}$ \\
\hline Darkest Red & $\because$ & Early-mid. & M. Martin Cahuzac & Semi-double & $\mathrm{xx}$ & $\mathrm{x}$ & $\mathrm{X}$ \\
\hline $\begin{array}{l}\text { Light Pink } \\
\text { Red }\end{array}$ & $\begin{array}{l}x \\
\cdots\end{array}$ & $\begin{array}{l}\text { Early-mid. } \\
\text { Very early }\end{array}$ & $\begin{array}{l}\text { Octavie Demay } \\
\text { Officinalis }\end{array}$ & $\begin{array}{l}\text { Crown } \\
\text { Semi-double }\end{array}$ & ${ }_{X}^{X X}$ & ${ }_{\mathrm{X}}^{\mathrm{x}}$ & $\frac{X X}{X}$ \\
\hline Deep Pink & $\ldots$ & Midseason & Petite Renee & Single & $\mathrm{XX}$ & $\mathrm{x}$ & ... \\
\hline Red & $\mathrm{Xx}$ & Midseason & Philippe Rivoire & Double & $\mathrm{X}$ & $x$ & .. \\
\hline Light Pink & $\mathrm{x}$ & Very late & Pierre Duchartre & Double & $\mathrm{x}$ & $\mathrm{x}$ & $\cdots$ \\
\hline Light Pink & $\mathrm{xx}$ & Late & President Wilson & Double & $\mathrm{x}$ & $\mathrm{X}$ & $\mathrm{xx}$ \\
\hline Pale Yellow & $\mathrm{XX}$ & Midseason & Primevere & Bomb & $\mathrm{X}$ & $\mathrm{x}$ & $\mathrm{X}$ \\
\hline Light Pink & $\mathrm{X}$ & Midseason & Reine Hortense & Semi-double & $\mathrm{X}$ & $\mathrm{xx}$ & $\mathrm{xX}$ \\
\hline Red & $\mathrm{xX}$ & Very early & Richard Carvel & Bomb & $\mathrm{XX}$ & $\mathrm{xx}$ & $x x$ \\
\hline Light Pink & $X$ & Midseason & Rose Shaylor & Double & $\mathrm{xx}$ & $\mathrm{xx}$ & $x x$ \\
\hline Light Pink & $\mathrm{X}$ & Late-mid. & Sarah Bernhardt & Semi-double & $\mathrm{xx}$ & $\mathrm{xX}$ & $\mathrm{Xx}$ \\
\hline Light Pink & $\mathrm{X}$ & Early & Simonne Chevalier & Crown & $\mathrm{XX}$ & $\mathrm{XX}$ & $\mathrm{XX}$ \\
\hline Cream White & $\mathrm{X}$ & Midseason & Solan & Double & $\mathrm{XX}$ & $\mathrm{xx}$ & $\mathrm{XX}$ \\
\hline Pale Yellow & $\mathrm{x}$ & Midseason & Solfatare & Bomb & $\mathrm{x}$ & $\mathrm{x}$ & $\mathrm{x}$ \\
\hline Red & $\cdots$ & Midseason & Souv. l'Ex. Bordeaux & Bomb & $\mathrm{xx}$ & $\mathrm{x}$ & \\
\hline Deep Pink & $\cdots$ & Midseason & Souv. de Louis Bigot & Double & $\mathrm{x}$ & $\mathrm{x}$ & $\mathrm{xx}$ \\
\hline White & $\mathrm{X}$ & Early-mid. & The Bride & Single & $\mathrm{X}$ & $\mathrm{xx}$ & $\mathrm{x}$ \\
\hline Light Pink & $\mathrm{X}$ & Early & Therese & Double & $\mathrm{xx}$ & $\mathrm{xX}$ & $\mathrm{XX}$ \\
\hline Light Pink & $\cdots$ & Midseason & Tokio & Japanese & $\mathrm{xx}$ & $\mathrm{x}$ & \\
\hline Light Pink & $\mathrm{x}$ & Late-mid. & Tourangelle & Double & $\mathrm{x}$ & $\mathrm{xx}$ & $\mathrm{XX}$ \\
\hline Light Pink & $\mathrm{X}$ & Midseason & Triomphe l'Ex. de Lille & Double & $\mathrm{XX}$ & $\mathrm{xx}$ & $\mathrm{xx}$ \\
\hline Light Pink & $\mathrm{X}$ & Very early & Umbellata Rosea & Double & $\mathrm{xx}$ & $\mathrm{x}$ & \\
\hline Light Pink & $\mathrm{X}$ & Midseason & Van Dyck & Crown & $\mathrm{XX}$ & $\mathrm{x}$ & \\
\hline Deep Pink & $\cdots$ & Midseason & Walter Faxon & Double & $\mathrm{XX}$ & $\mathrm{XX}$ & $\mathrm{XX}$ \\
\hline
\end{tabular}

See pages 21 to 40 for detailed descriptions and prices. 


\section{Types of Bloom}

Paeonia albiflora, or P. Chinensis, as it is more commonly catalogued, has by the American Peony Society been divided into seven types as follows:

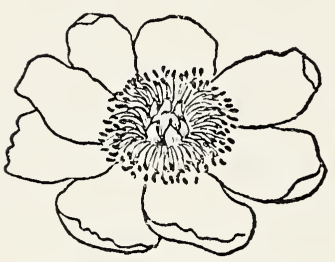

SINGLE. Those with a single row of wide guards and a center of yellow pollen-bearing stamens.

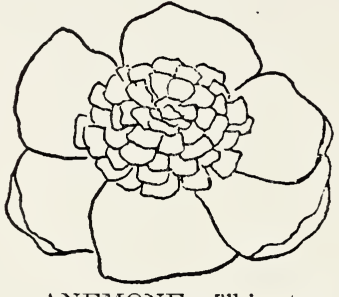

ANEMONE T'his type is a step further in the process of doubling, with the stamens all transformed into short narrow petals, forming a round cushion in the center of the flower.

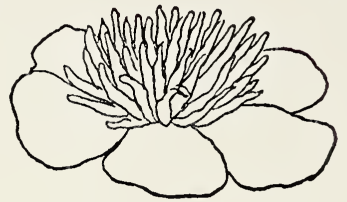

JAPANESE. These have wide guards the same as the Singles, but with the stamens and anthers greatly enlarged into narrow, thick petaloids of various colors, tipped with vestiges of the yellow anthers without pollen.

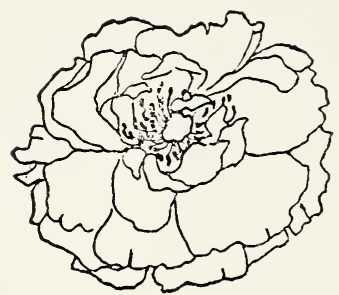

SEMI-DOUBLE. Those with several rows of wide petals and a center of stamens and partially transformed petaloids. Many of the reds are of this type.

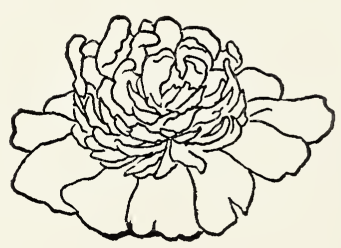

CROWN. In this type wide petals are developed in the center of the flower, forming a high crown with the narrow, short petals forming a ring or collar around it. Often the crown and guards are one color, and the collar another or lighter shade.

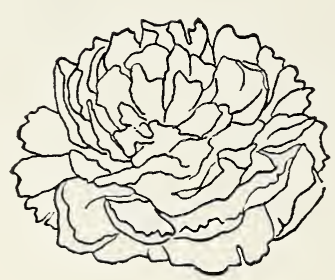

DOUBLE. In these the process of doubling is completed, all stamens fully transformed into evenly arranged wide petaloids, similar to the guards, forming a perfect rose-shaped bloom.

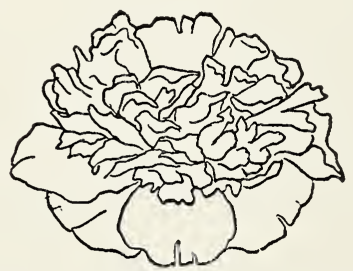

BOMIB. The next step is this type in which all the center petals are uniformly wide approaching the guards, but distinctly differentiated from them, forming a globe-shaped center without collar or crown. 


\title{
Descriptive Price List
}

\author{
T parenthesis we give the name of the originator and date of \\ introduction, followed by American Peony Society Symposium \\ rating. Color names in italics are from Ridgway's Color Stan- \\ dards and Nomenclature.
}

ADOLPHE ROUSSEAU (Dessert \& Mechin 1890 ; 8.5). Very large, dark, pomegranate purple with metallic reflex. Classed as semi-double but on old, established plants, which grow very tall, the flowers vary in form from almost single to semi-double. Among the earliest reds, its striking appearance makes it very much in demand. Two-year, $\$ 3.00$. Divisions, $\$ 2.00$.

ALBERT CROUSSE (Crousse 1893; 8.6). One of the most striking and perhaps the most distinct Peony in existence, there being nothing else of just the same form and character. Immense bloom, very full and compact; small, imbricated and very dense petalage from the outside to the very heart of the flower. Rose pink, with centre shaded clear pink. In color and form like an enormous pink carnation. Very sweet fragrance. Tall, erect in growth and a late bloomer. A superb and an extraordinary Peony. Two-year, $\$ 1.50$. One-year, $\$ 1.25$. Divisions, $\$ 1.00$.

ALEXANDRIANA (Calot 1856; 7.1). Very large and full double rose pink with silver edges. An unusually strong grower, with great quantities of delightfully fragrant flowers on strong upright stems. A very fine early cut-flower sort. In spite of its comparatively low price, one of the most valuable Peonies we have. Two-year, $\$ 1.10$. One-year, 95c. Divisions, 75c.

ALICE DE JULVECOURT (Pele 1857; 7.2). Very double, perfectly built fragrant flower; soft pink, delicately shaded rose and cream, with central petals beautifully edged with red. An exquisite combination of coloring not easily described. A very free and rather early bloomer. (We consider this one of the very finest Peonies of the light varieties in cultivation). Two-year, $\$ 1.10$. One-year, 95c. Divisions, 75c.

ALSACE LORRAINE (Lemoine $1906 ; 8.8$ ). This tall and vigorous plant carries erectly large, white semi-rose type flowers which bloom in late mid-season. The white petals are pointed and reflect the light of the stamens, suggesting the water lily. Divisions only, $\$ 3.00$.

ASA GRAY (Crousse 1886; 8.1). Immense, massive and compact bloom; imbricated like a rose; very full and fine of form. Pale rose pink very delicately dotted with carmine. An imposing and extraordinary flower and very fragrant. Two-year, \$3.00. Divisions, $\$ 2.00$.

AURORE (Dessert 1904; 8.0). A fragrant cup-shaped flesh pink flower with petals loosely arranged in semi-rose form. Prominent yellow stamens add a cheerful light to the collar petals, while flecks of crimson are seen on the wide center petals. It opens in mid-season on a medium-tall plant; is often employed for landscape effects. Two-year, $\$ 1.50$. One-year, $\$ 1.25$. Divisions, $\$ 1.00$.

AVALANCHE (Crousse 1886; 8.7). Large, glorious bloom

TWELVE OF ANY ONE VARIETY FOR THE PRICE OF TEN

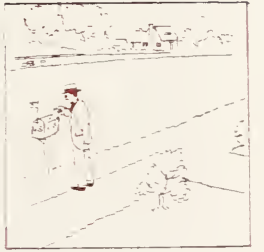

Easy to Order 


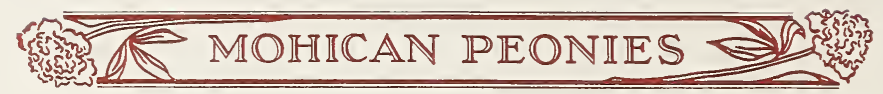

of perfect form; fine, milk white; creamy center. Absolutely identical with Albatre. The fragrant flowers bloom abuntantly in late mid-season and are fine for cutting. Two-year, $\$ 1.50$. Divisions, $\$ 1.00$.

BARONESS SCHROEDER (Kelway 1889; 9.0). Flesh changing to white, and a free bloomer. Exceedingly rare. One of the finest old England has produced. Two-year, $\$ 2.25$. Divisions, $\$ 1.50$.

BOULE DE NEIGE (Calot 1867; 8.0). Would you like to prolong the period of bloom of Festiva Maxima-and keep it sweetly fragrant at the same time? If so, plant this strong grower of large, white, crimsonflecked flowers, often used for landscape work and always a satisfactory cut flower. Two-year, $\$ 1.50$. Divisions, $\$ 1.00$.

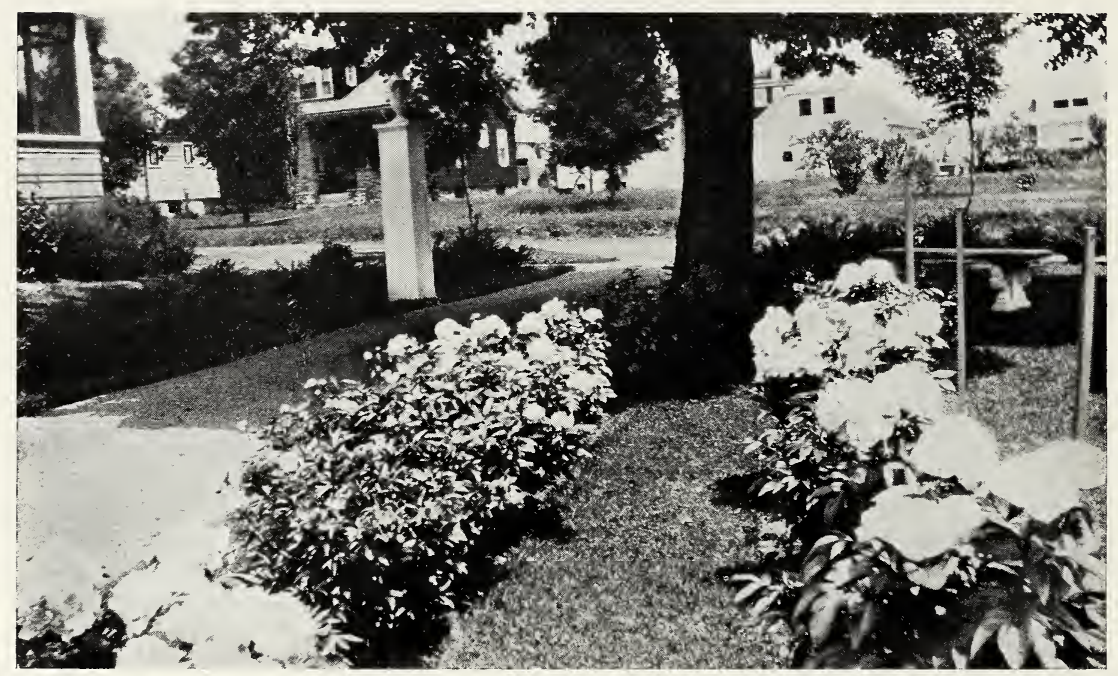

Mohican's Challenge Collection-Submitted by Charles Bissell, Providence, R. I. Honorable Mention.

CHARLES VERDIER (Origin unknown). Wonderfully fragrant are the very dark rosalane purple flowers which bloom abundantly on a mediumheight compact plant. Because of its unusual color and its fragrance it requires no courage to persist in offering this Peony although it is unrated. The uncertainty of its origin makes it the more interesting. Two-year, $\$ 3.00$. Divisions, $\$ 2.00$.

CHERRY HILL (Thurlow $1915 ; 8.6$ ). Very early red with medium sized double blossoms on very tall strong stems. The deep, rich maroon blossoms have a glistening sheen and show yellow stamens. Excellent for cutting. Divisions only, $\$ 5.00$.

CLAIRE DUBOIS (Crousse 1886; 8.7). One of the very largest Peonies in cultivation. Immense, solid, globular. Center very full, convex, and tufted; petals incurved and fringed. Exquisite clear, rose pink, with a satiny reflex, deeper in color at center. Very tall and a rare bloomer,

TWELVE OF ANY ONE VARIETY FOR THE PRICE OF TEN 


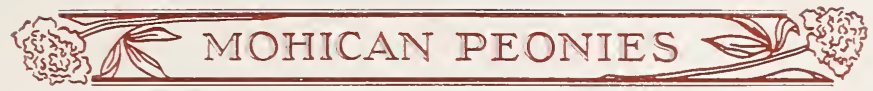

fragrant. A superb impressive flower. Two-year, \$2.25. One-year, \$1.85. Divisions, $\$ 1.50$.

CONSTANT DEVRED (Calot 1868; 7.5). This striking, rose-type, red is especially desirable because it is the last of the reds to bloom. The full, compact carmine-red flowers are held erect on the long, stiff stems of a vigorous plant. These characteristics make it a valuable cut flower. Two-year, \$1.50. Divisions, $\$ 1.00$.

COQUELIN (Dessert 1905; 7.2). One of the very earliest varieties to bloom, providing an abundance of color early in the season. The large, cup-shaped flower of the semi-double type has imbricated petals of brilliant spinel pink, tipped with silver, shading to cameo with amber stamens. Two-year, \$1.10. Divisions, 75c.

COURONNE D'OR (Calot $1873 ; 8.1$ ). A late, exceedingly fragrant, ballshaped bloom. Snowy white with yellowish reflex and carmine edges on center petals. Incomparably lovely and one of the very choicest and best Peonies in cultivation. Two-year, $\$ 1 .-$ 50 . One-year, $\$ 1.25$. Divisions, $\$ 1.00$.

DORCHESTER (Richardson $1870 ; 7.7$ ). Beautiful and delicate salmon flesh; a well-built and striking variety. $\mathrm{Ra}$ ther a dwarf grower, but blooms come on strong, upright stems. Very late, coming in after most other varieties have passed. Twoyear, $\$ 1.50$. Divisions, $\$ 1.00$.

DUCHESSE DE NEMOURS ( $\mathrm{C}$ alot $1856 ; 8.1)$. An early white of the crown type opening in a globular, cupped form that is very distinct. The color is clear white, with a collar of sulphur white, and a greenish reflex at the center that is most refreshing. It is a vigorous grower, of medium

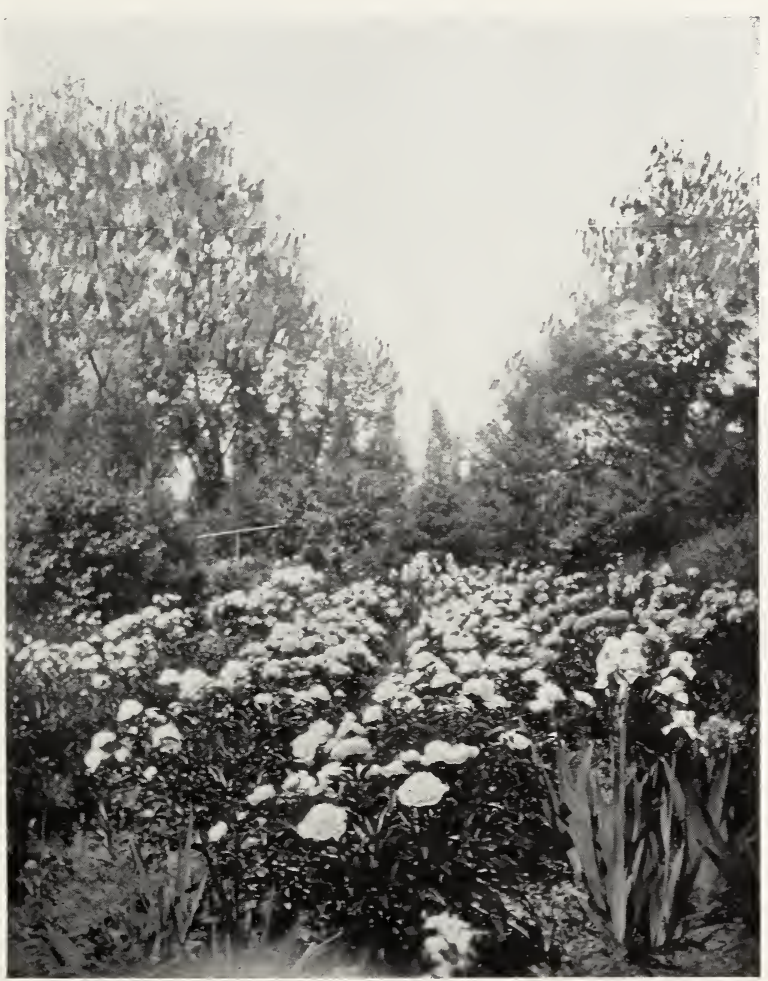

Edulis Superba-Submitted by Miss Florence E. Beck, Camp Hill, Penna. Honorable Mention.

TWELVE OF ANY ONE VARIETY FOR THE PRICE OF TEN 


\section{MOHICAN PEONIES}

height, a free bloomer, and fragrant. A useful variety, either for the garden or for commercial cutting. This is another one of the older varities that is outstanding, even when compared with all the newer and more expensive introductions. Two-year, $\$ 2.25$. Divisions, $\$ 1.50$.

DUKE OF WELLINGTON (Calot 1859; 7.8). A sturdy, tall-growing plant that supports its enormous white flowers with graceful ease. The fragrant bomb-shaped flowers have broad guard petals of pure white surrounding a spherical center of delicate citron yellow. It is so well constructed that it makes an excellent show flower, comparing favorably with the very best of the whites. A reliable midseason cut flower. Two-year, $\$ 2.25$. Divisions, $\$ 1.50$.

EDULIS SUPERBA (Lemoine $1824 ; 7.6$ ). Very beautiful, large, full and well-formed bloom, with good culture measuring from seven to eight inches across. Brilliant deep rose pink with silvery reflex. A vigorous grower producing quantities of very fragrant flowers on strong stems. In this climate it rarely fails to be ready on Memorial Day. It is really one of the most valuable Peonies because of its extreme earliness, together with the fact that it leads the field in fragrance, in color, in vigor and in durability as a cut flower. Two-year, $\$ 1.50$. One-year, $\$ 1.25$. Divisions, $\$ 1.00$.

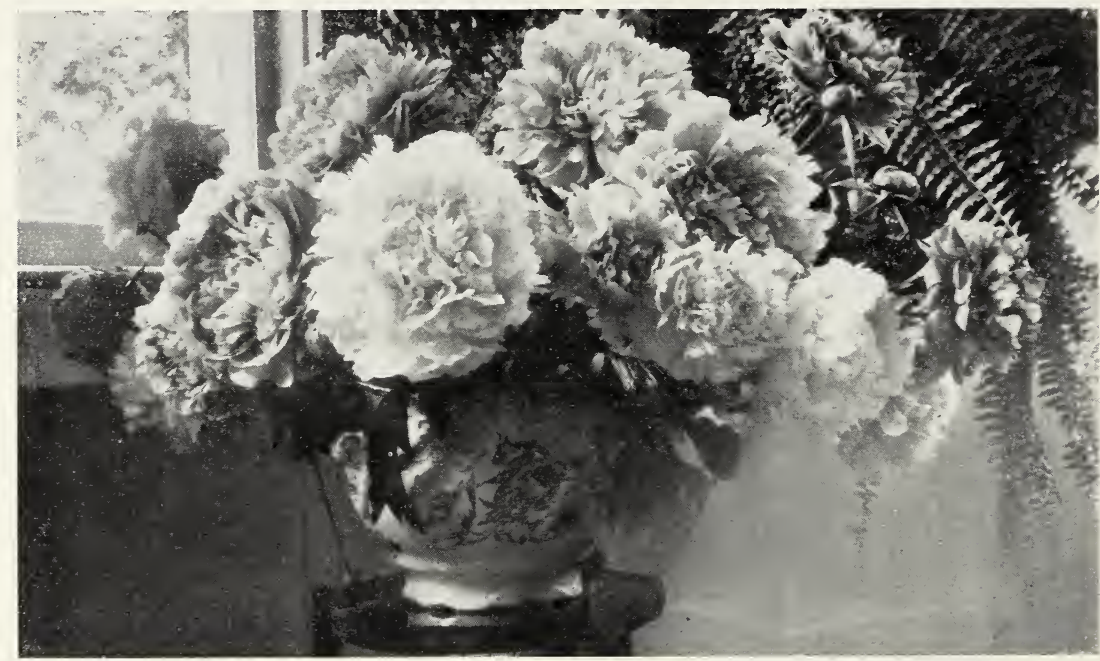

Edulis Superba, Felix Crousse and Festiva Maxima-Submitted by A. J. Parks, Syracuse, N. Y. Honorable Mention.

EDWIN C. SHAW (Thurlow 1919; 9.1). A clear, shell pink; outer petals broad and rounded, sometimes slightly edged crimson. Translucent texture. The petals are nearly the same length except the extreme center petals which are short. This gives a very pretty cup-shape and the general effect is one of elegance of form. Late mid-season. Delicious fragrance. Divisions only, $\$ 20.00$.

TWELVE OF ANY ONE VARIETY FOR THE PRICE OF TEN 


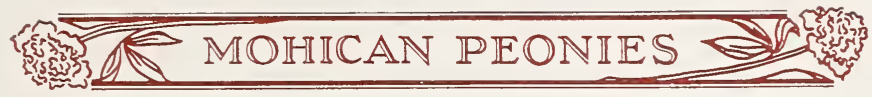

EMPEROR NICHOLS (Crousse 1897; 7.1). This vigorous bomb-shaped red is one of the most distinct varieties in our Gardens. The brilliance of this dark amaranth purple Peony commands the admiration of those who like colorful flowers. Although not of the same tone nor the same shape it serves as a delightful follower for the justly popular Felix Crousse, blooming after Felix Crousse has "gone by." We of Mohican Gardens are very fond of Emperor Nicholas. Two-year, $\$ 3.00$. Divisions, $\$ 2.00$.

EUGENE REIGNOUX (Dessert 1905; 7.3). This large, strong-growing variety produces, during mid-season, a profusion of cup-shaped semidouble flowers of a deep rose pink. Two-year, 75c. Divisions, 50c.

EUGENE VERDIER (Calot 1864; 8.3). Very large, compact globular bloom of deep rose pink, deeper toward the center. Vigorous, profuse and delicately fragrant. Excellent for cutting. This variety is one of the latest to bloom and is at its best in a cold climate. Divisions only, $\$ 2.00$.

EUGENIE VERDIER (Calot $1864 ; 8.6$ ). Here is a most desirable, fragrant mid-season pink. It is an excellent cut flower, delicately hydrangea colored, and of fine texture. The outer petals of this semirose type are of lighter shade than the center. Its large size and delicate coloring make it an excellent show flower. Divisions only, $\$ 1.50$.

\section{FELIX CROUSSE}

(Crousse 1881 ; 8.4). Typical bombshaped red, with ruby-flamed center. Fairly dazzling in the brilliancy of its distinct color. It is in fact, the greatest red Peony in cultiva. tion, and should be included in every collection. Free mid-season bloomer, and quite fragrant. Very similar but decidely superior to Augustin d'Hour, which variety we do not offer for that reason. Twoyear, $\$ 1.50$. Oneyear, \$1.25. Divisions, $\$ 1.00$.

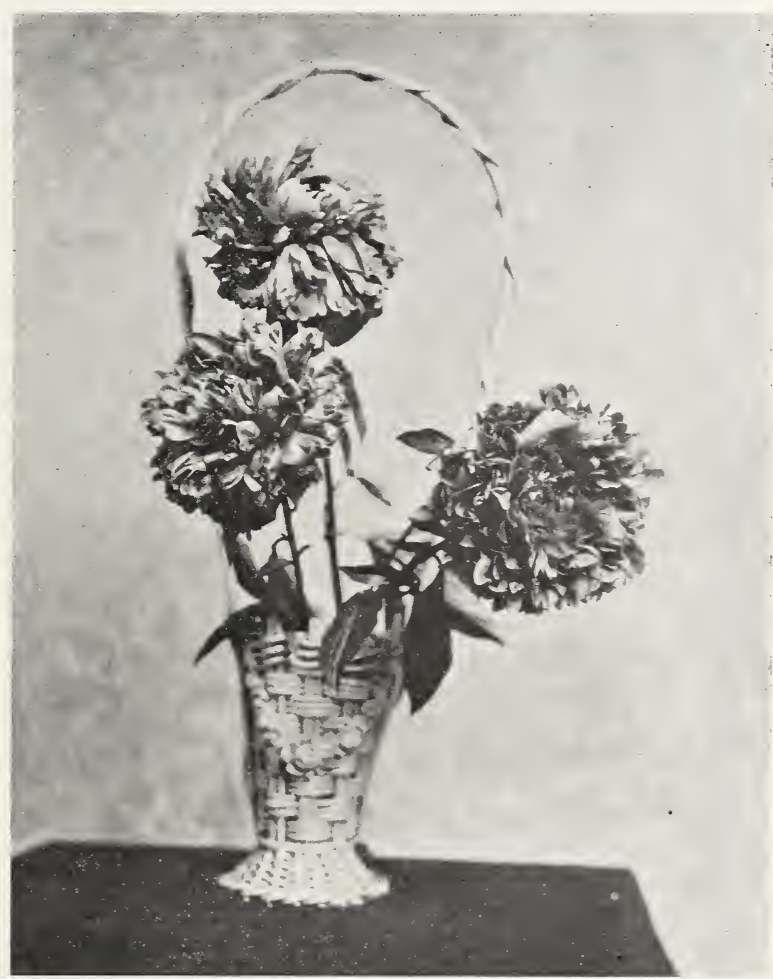

Felix Crousse-Submitted by Mrs. Emma Gehl, Traer, Iowa. Honorable Mention.

TWELVE OF ANY ONE VARIETY FOR THE PRICE OF TEN 
FESTIVA MAXIMA (Miellez 1851; 9.3). Very large and full, pure white flower, with few center petals tipped with blood-red spots. A vigorous grower, with massive foliage and long stiff stems. Fragrant, early and free bloomer. Two-year, $\$ 1.50$. One-year, $\$ 1.25$. Divisions, $\$ 1.00$.

FLORAL TREASURE (Rosefield 1900; 7.5). The large double flowers are flesh colored in the center with pale rhodonite pink guards. A vigorous plant, a wonderful late mid-season bloomer, an excellent cut flower. An American production of very great merit and a splendid "hemisphere of fragrance." Two-year, \$1.50. One-year, \$1.25. Divisions, $\$ 1.00$.

GERMAINE BIGOT (Dessert $1902 ; 8.5$ ). One of Dessert's finest triumphs. The flowers are large and compact, of the crown type. The color is cameo pink, with lighter flesh colored tints in the center; the large guards are splashed with crimson. The plant produces an abundance of flowers, in clusters, during midseason. Two-year, $\$ 1.50$. Divisions, $\$ 1.00$.

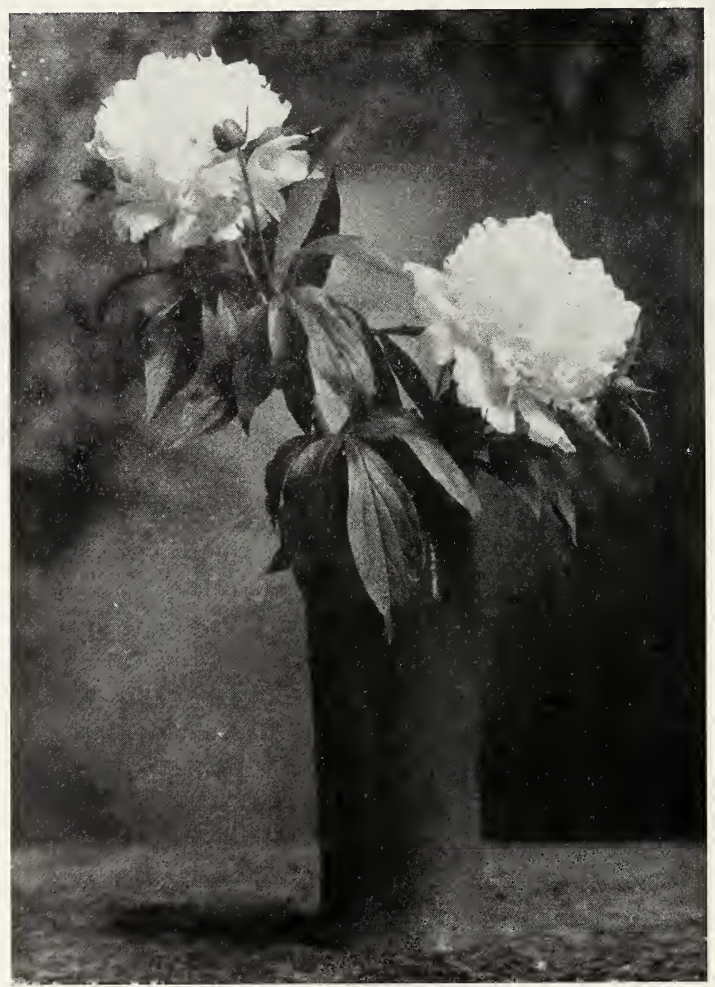

Festiva Maxima-Submitted by Mrs. M. B. Sprigg, Weston, W. Va. Honorable Mention.
GLOIRE DE CHARLES GOMBAULT (Gombault $1866 ; 7.9$ ). This Peony is distinctly first and foremost of the multicolor varieties. It easily surpasses them all, and standing upon its own merits we consider it one of the most strikingly beautiful varieties on our list. Large, globular flowers, extra dense and compact. Deep flesh pink collar; narrower center petals of salmon and apricot, touched here and there with bright carmine. Fragrant mid-season bloomer on extra long stout stems. Ideal for cut flowers. Two-year, $\$ 1.50$. One-year, \$1.25. Divisions, $\$ 1.00$.

GRANDIFLORA (Richardson 1883 ; 8.8). Exquisite rhodonite pink, with somewhat lighter shades in center. Base of petals touched with straw yellow reflecting upward through the blossom in salmon shades. One of the 

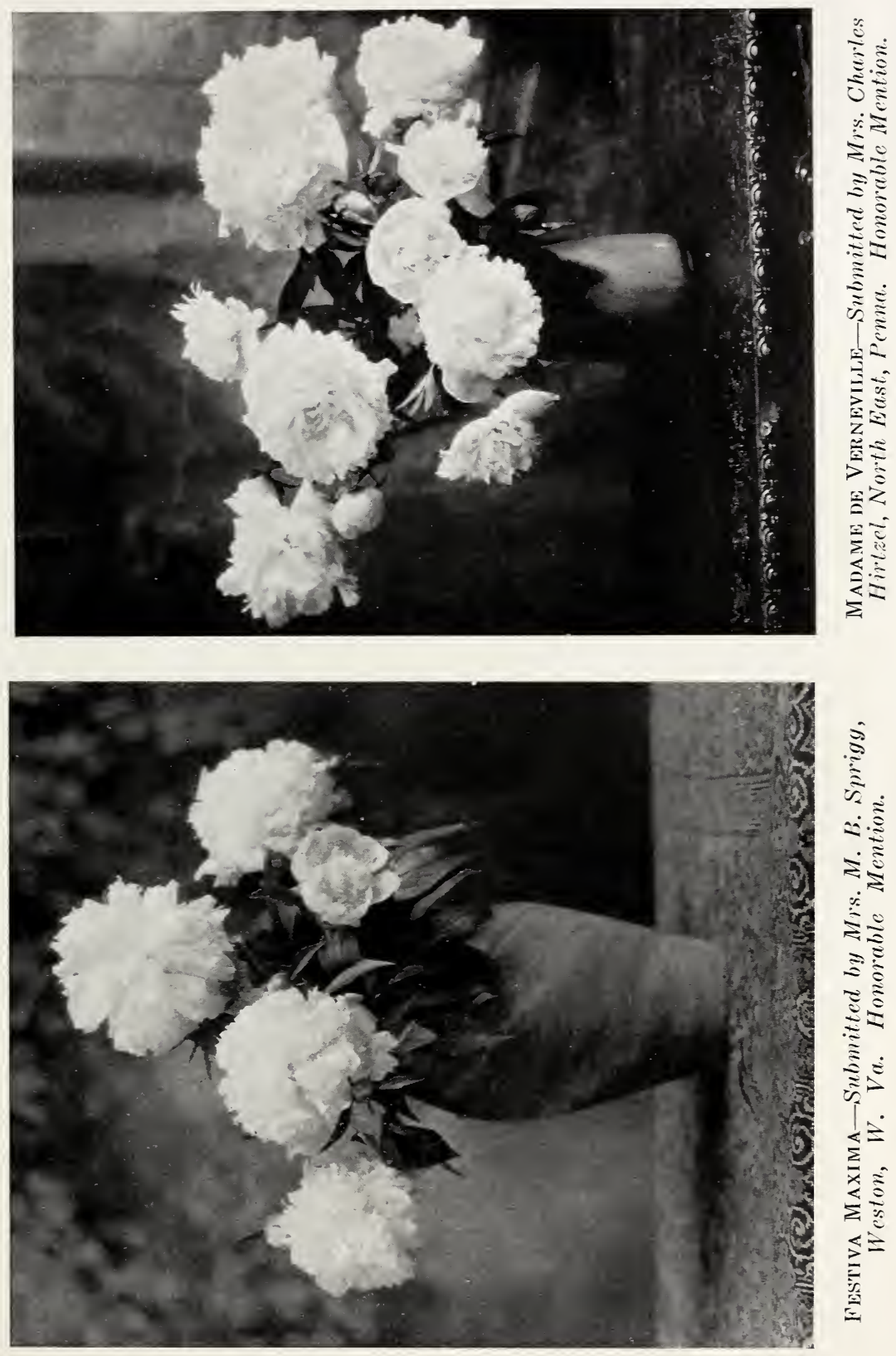


\section{MOHICAN PEONIES}

very largest Peonies and the latest pink to bloom. Fragrant. Divisions only, $\$ 2.00$.

GRANDIFLORA NIVEA PLENA (Lemoine 1824; 8.1). A very early and superb Peony. Large and full; pure white collar; center, shaded salmon and sulphur. Fine. Divisions only, $\$ 1.50$.

JEANNE D'ARC (Calot 1858; 7.9), A glorious flower-a very "splendor of fragrant loveliness." The collar is yellow; the guards.pale rhodonite pink and the full, high center a delicate pink with occasional carmine spots. Exquisite and, with the exception of Gloire de Charles Gombault, the very finest tri-color yet produced. Free, mid-season bloomer. Two-year, \$1.50. One-year, $\$ 1.25$. Divisions, $\$ 1.00$.

JUDGE BERRY (Brand 1907; 8.6). This early double pink opens its long petals to an immense dise of fragrant loveliness. Even young plants blossom freely on the medium tall stems. Divisions only, $\$ 6.00$.

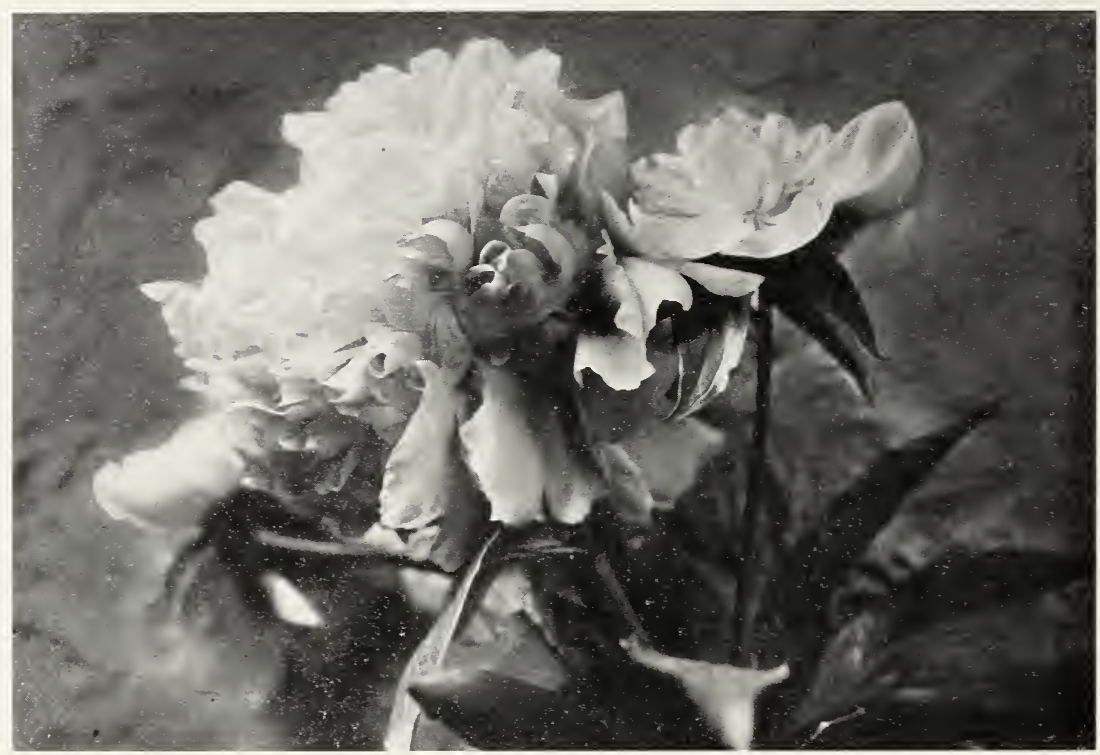

Gloire de Charles Gombault-Submitted by Mrs. Annie Blessing, Reading, Penna. Awarded one of Seven Prizes.

KARL ROSEFIELD (Rosefield 1908; 8.8). Rich, clear, glowing crimson. Semi-double type flower of fine form. Full double, large and solidly built. Strong erect grower and good sure bloomer. Very good as a keeper when cut and has no superior as a cut-flower red. One of the very best reds for any purpose. Two-year, $\$ 3.00$. One-year, $\$ 2.50$. Division $\$ 2.00$.

KELWAY'S GLORIOUS (Kelway $1909 ; 9.8$ ). It is generally agreed that only Le Cygne, the finest of them all, exceeds the beauty of Kelway's Glorious. The white flower opens in mid-season to a large, full, double of wonderful fragrance. Unless
to be used for cutting, the plant should not be disbudded beTWEIVE OF ANY ONE VIRIETY FOR THE PRICE OF TEN 


\section{MOHICAN PEONIES}

cause of the prolonged season resulting from the opening of the laterals. An excellent Peony for landscape effect. One year, \$15.00. Divisions, $\$ 12.00$.

LADY ALEXANDRA DUFF (Kelway 1902; 9.1). Unusual, large, flat, loosely assembled flowers with petals irregular in size and shape. Delicate amaranth pink guard petals. Very pale pink, almost white, are the other petals in this cup-shaped flower. Yellow stamens repose boldly within the cup. To see it is to test its delicious fragrance. Two-year, $\$ 4.50$. Divisions, $\$ 3.00$.

LAFAYETTE (Dessert 1904; 7.8). The large, fragrant flowers are unique in color, being Tyrian pink with touches of heliotrope and a delicate tinting of cerise in the reflex. The petals are beautifully fringed. The flowers bloom in mid-season and last well for cutting. Lafayette is a very pleasing and desirable flower. Two-year, $\$ 1.50$. Divisions, $\$ 1.00$.

LAMARTINE (Calot $1860 ; 8.3$ ). This is an enormous compact bloom of the double type. It is rose-pink and possesses a delightful fragrance. Blooming freely in mid-season, it is a fine variety for garden display and has good keeping qualities. Two-year, $\$ 2.25$. Divisions, $\$ 1.50$.

LA PERLE (Crousse 1885; 8.5). This is indeed a gem, well named by its famous originator. Pale rose-pink, with a touch of lilac-pink on the edges of the petals, which fade to almost pure white when fully open. A profusion of fragrant, double blooms seen near the end of the season tempts the visitor to find room for this delightful Peony. Two-year, $\$ 1.50$. Divisions, $\$ 1.00$.

LA ROSIERE (Crousse 1888; 8.3)). This is not a yellow flower but its center of golden yellow stamens surrounded by cream white tempts the use of "yellow" for accurate description. In form and effect it resembles a large tea rose with broad, imbricated petals. It is double in type. The very fragrant flowers open in mid-season, growing in profuse clusters and creating a delightful effect if not disbudded. Very satisfactory as a cut flower. Two-year, $\$ 2.25$. Divisions $\$ 1.50$.

LA TENDRESSE (Crousse 1896; 8.1). Very full, immense flowers with thick, wax-like petals. Color creamy-white changing to pure white, flecked with crimson. Slightly fragrant, blooming in clusters during early midseason. Two-year, $\$ 1.50$. Divisions $\$ 1.00$.

LA TULIPE (Calot 1872; 7.5). (See illustration page 2 ). This is the Peony that tulips strive to resemble. It is a pale rose pink with flesh tints and, on the center petals, touches of red. The fragrant blooms open late, on tall sturdy stems. It is a good cut flower. Two-year, $\$ 1.50$. Divisions, $\$ 1.00$.

LAURA DESSERT (Dessert 1913; 8.8). Anemone type; early mid-season; medium size and height. Guards pale pink in bud, opening pale lemon, fading white; center a flat, compact tuft of petaloids deep cream at base, paling outward giving a light lemon yellow effect. Moderately fragrant. Free blooming on sturdy upright stems. Two-year, \$11.25. One-year, $\$ 9.35$. Divisions, $\$ 7.50$.

LE CYGNE (Lemoine 1907; 9.9). People who have seen Le Cygne never have to ask why it is rated the highest of all Peonies. It is a perfect flower, perfect in its whiteness, its form, its texture, its fragrance, its size, and above all vigorous and requiring only the same care that is given to any ordinary plant. Two-year, $\$ 15.00$. One-year, $\$ 12.50$. Divisons, $\$ 10.00$.

TIVELIE OF ANY ONE VARIETY FOR THE PRICE OF TEN

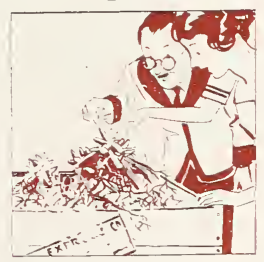



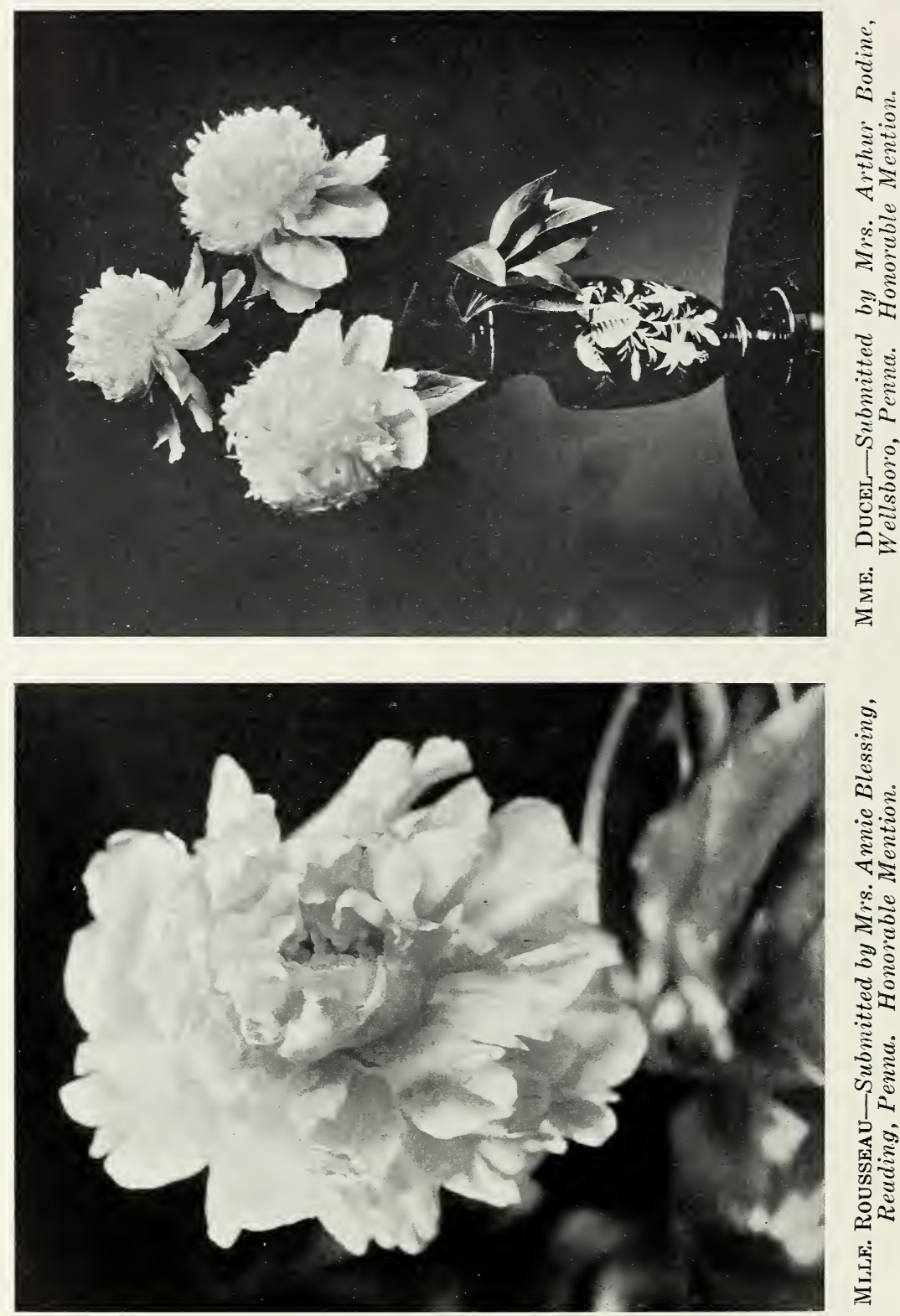


\section{MOHICAN PEONIES}

LIVINGSTONE (Crousse 1879; 8.1). Enormous, very massive, imbricated and compact bloom. Pale lilac-rose with silver tips; center petals flecked with carmine. A vigorous grower and one of the latest to bloom. Long, strong stems. Exquisite in the bud and a great keeper but lacking in fragrance. A Peony of the highest class. One-year, \$1.25. Divisions, $\$ 1.00$.

LONGFELLOW (Brand 1907; 9.0). The rich red of this gorgeous Peony always assures it a prominent place in any garden and is sure to win the affections of those who love red Peonies. The brilliant cherry-red color is not affected by sunshine as are some of the other red varieties. Divisions only, $\$ 5.00$.

MADAME AUGUSTE DESSERT (Dessert 1899; 8.6). Large, imbricated, cup-shaped flower, perfect in form with rounded petals of pale rose pink tinged with aster purple. Holds itself together neatly and with "sassy" diginity. The fragrant flowers bloom freely, during early mid-season.

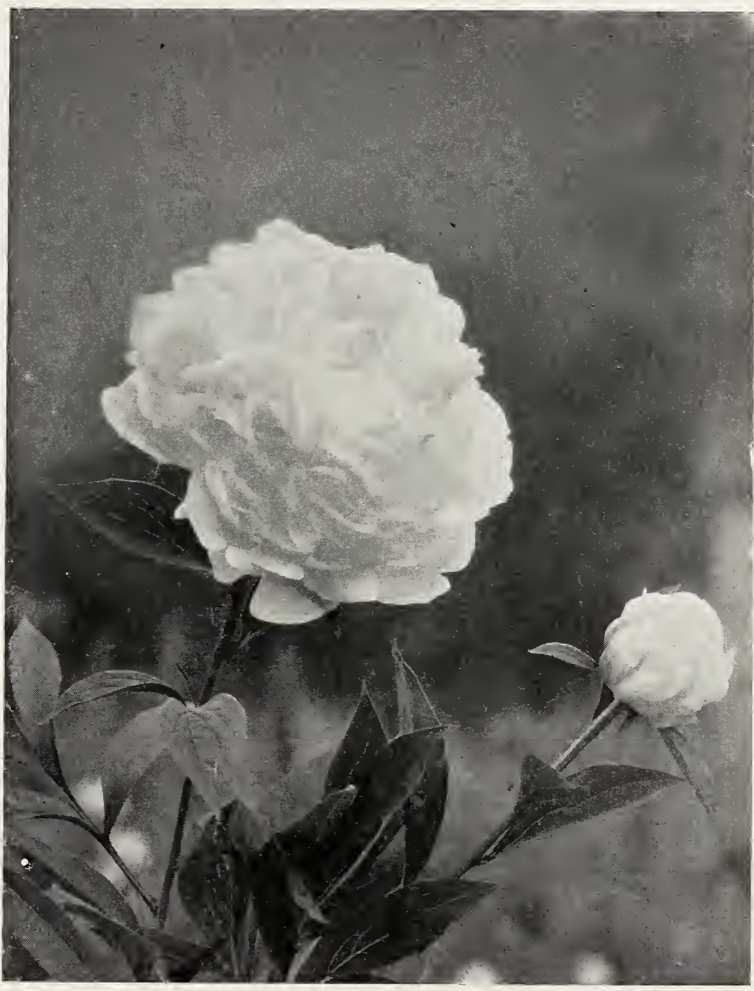

Marguerite Gerard-Submitted by Mrs. Frank Pagan, Wellsboro, Penna. Honorable Mention. Pagan, Wellsboro, Penna. Honorable Mention. cutting or for garden
TWELVE OF ANY ONE VARIETY FOR THE PRICE OF TEN
Two-year, \$1.50. Di-

MADAME BUCQUET Dessert $1888 ; 7.3)$. A magnificient flower-a striking and remarkable dark peony, and one of the best of the black sorts. Velvety black amaranth purple with garnet reflections. Medium in size, fragrant, strong grower, and free bloomer. Indispensable. One-year $\$ 1.85$. Divisions $\$ 1.50$.

MADAME CALOT (Miellez 1856; 8.1). Large, well-built rose type flower. Pale pink with darker center; collar tinged with silver, Prominent carpels add a touch of pink. The fragrant flowers bloom in early-mid-season. Two-year, \$1.50. Divisions, $\$ 1.00$.

M M E. C A M I L L E BANCEL (Crousse $1897 ; 7.9$. This fragrant light pink has large well-formed flowers which are very satisfactory for visions, $\$ 1.00$. 


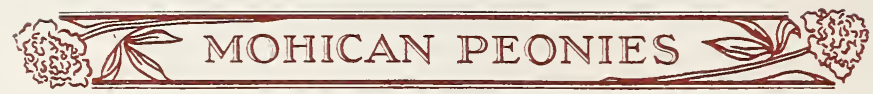

decoration. The distinctive double flowers open late in the season. Two-year, \$1.50. Divisions, $\$ 1.00$.

MADAME CROUSSE (Calot 1866; 7.9). Enormous, very double, lateblooming, paper white, slightly edged in center with carmine. Very fragrant and one of the best whites we have for cut flowers. Always distinct in a garden of many Peonies. Divisions only, $\$ 1.50$.

MADAME DE GALHAU (Crousse 1883; 7.5). This is perhaps the loveliest of the late bloomers. The flower is a large compact pale lilac-rose with rose-white guards, rather different and therefore worthy of more attention. Its lateness and its fragrance add to its charm. Two-year, $\$ 1.50$. Divisions, $\$ 1.00$.

MADAME DE VATRY (Guerin 1863; 7.7). We plead with our customers to give this lovely Peony a trial. Its fragrance is unusually attractive. Its three colors consist of cream-white collar, pale rose-pink guards and center touched with red. Two-year, \$2.25. Divisions, $\$ 1.50$.

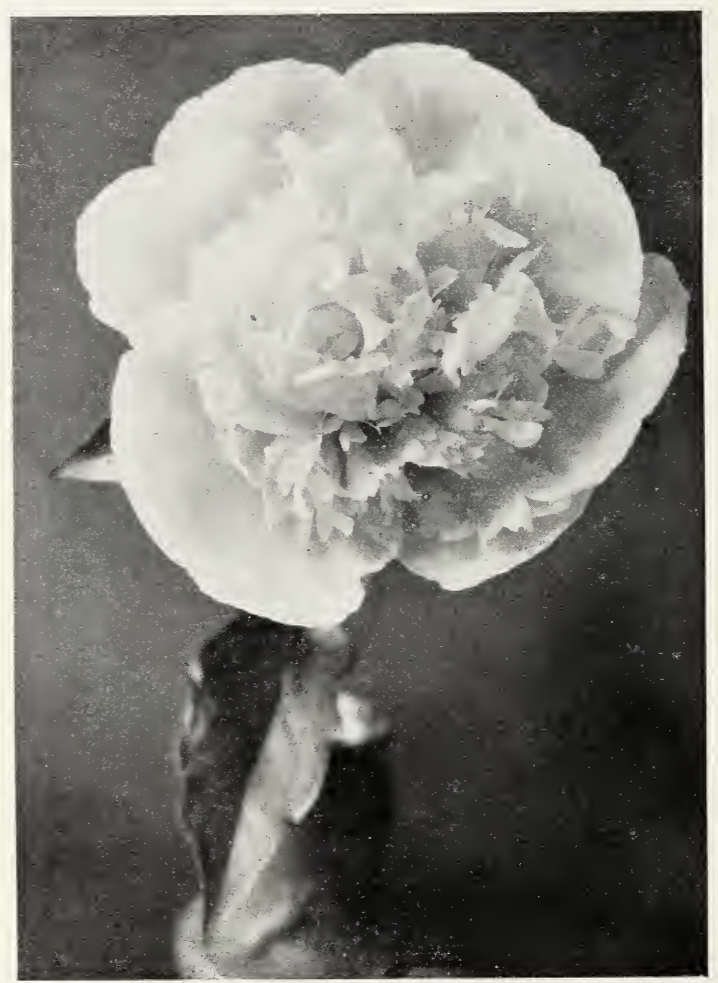

Madame de Verneville-Submitted by $D$. $W$. Morden, Pilot Mound, Manitoba, Canada. Honorable Mention.

MADAME DE VERNEVILLE (Crousse $1885 ; 7.9)$. Verylarge, exquisitely beautiful imbricated, bombshaped white. The guard petals are pure paper white and very broad, folding over the flowers; center daintily suff used with blush and cream tipped carmine. The whole flower heavy and compact. Strong grower, a free bloomer and early with a delightful sweet rose like fragrance. Oneyear, $\$ 1.25$. Divisions, $\$ 1.00$.

MADAME DUCEL (Mechin 1880; 7.9). Enormous, globular and compact bloom; literally packed with petals, which are somewhat incurved like chrysanthemums. A striking, brilliant shade of deep rose pink with silvery reflex. Holds its form and color to the end. Considered by many the very finest formed pink in existence. A magnificient cut-

TWELVE OF ANY ONE VARIETY FOR THE PRICE OF TEN 


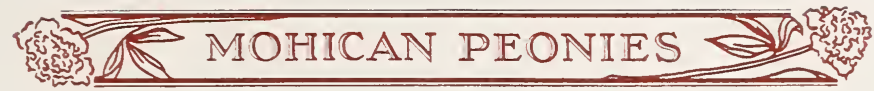

flower variety and wonderfully effective for landscape work. Stands among the very first on our "Challenge" list. Fragrant and early. Oneyear, $\$ 1.25$. Divisions, $\$ 1.00$.

MADAME EMILE GALLE (Crousse 1881; 8.5). Large, cup-shaped and imbricated bloom. Exquisite shade of soft lilac, with milk-white center which slowly changes to pronounced cream-color. A refined and rarely beautiful flower, not easy to describe. A very fragrant flower of double type, blooming freely rather late in the season. One-year, \$1.25. Divisions, $\$ 1.00$.

MADAME EMILE LFMOINE (Lemoine 1899; 8.9). Enormous and fragrant flower; white with creamy cast accentuated by the primuline yellow stamens. Red tipped seed pods give the effect of a pink center. Exceedingly rare. Divisions only, $\$ 2.00$.

MADAME FOREL (Crousse 1881; 7.7). This is a fragrant, late-blooming variety of striking effect. The strong stems and full ball-shaped flowers make it a good variety for cutting. In color, it is a clear deep rose pink. Divisions only, $\$ 1.00$.

MADAME GEISSLER (Crousse $1880 ; 7.9)$. One of the very largest Peonies in existence; very double, globular. Color, a glossy silver pale amaranth pink, shading to tyrian pink. at base of petals. Delicious and distinct fragrance. An abundant mid-season bloomer; heavy and perfect flowers coming even on the youngest plants. An ideal keeper, one of the best for cut flowers. True stock of this variety is very scarce. Twoyear, $\$ 1.50$. Oneyear, \$1.25. Divisions, $\$ 1.00$.

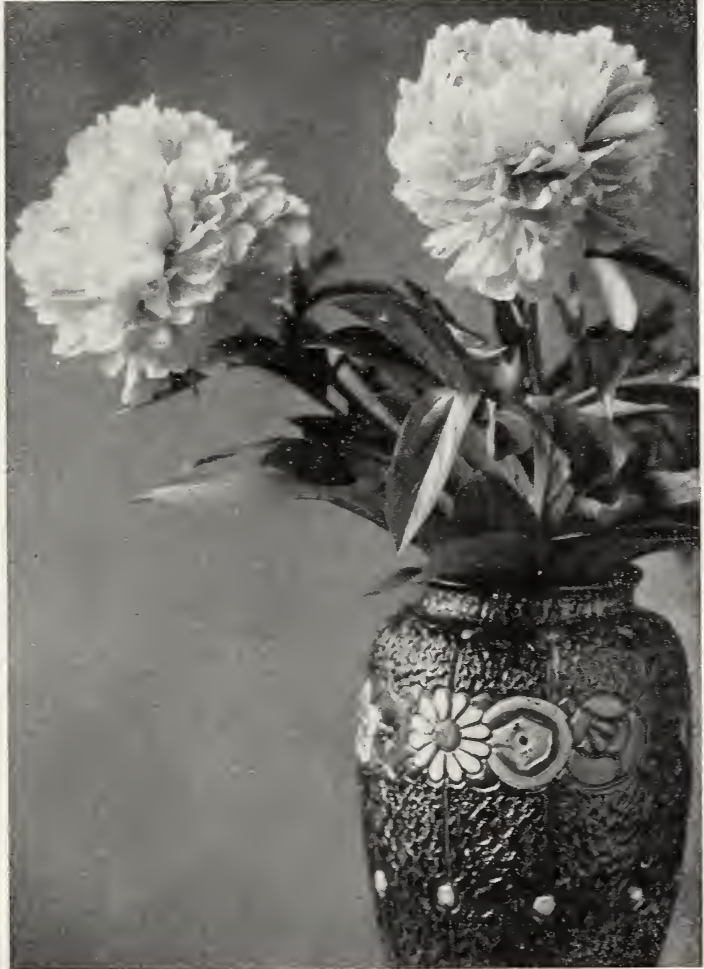

MADAME GeISSLER-Submitted by Fred E. Stevenson, Killarney, Manitoba, Canada. Honorable Mention.

\section{TWELVE OF ANY ONE VARIETY FOR THE PRICE OF TEN}


MADEMOISELLE ROUSSEAU (Crousse 1886; 8.1). This gorgeous fleshpink Peony blooms freely on tall, sturdy stems. The guard petals of this semi-double flower are splashed with carmine, as are some of the center petals. It is a fragrant mid-season Peony of first rank. Two-year, \$1.50. Divisions, $\$ 1.00$.

MARCELLE DESSERT (Dessert 1899; 8.2). Immense flowers of perfect crown form with the exquisite fragrance of a tea rose. The color is a glossy milk-white, splashed with lilac and with occasional crimson flecks in the center. Especially fine foliage. Very desirable. Two-year, \$2.25. Divisions, $\$ 1.50$.

MARGUERITE GERARD (Crousse 1892; 8.4). (See illustration on page 31). Fragrant, compact, semi-double developing into a crown with stamens. Broad, pale rhodonite-pink petals, changing to soft blush, almost white. Creamy white center. Exquisite free-flowering Peony and very rare. This Peony challenges description. Visitors at the gardens always order more than do our catalog customers. Two-year, $\$ 1.50$. Divisions, $\$ 1.00$.

MARIE CROUSSE (Crousse i892; 8.9). Exquisite, large, globular flower, very full. Soft, flesh pink, shaded with glossy lilac. Tall, erect and strong grower-free bloomer in mid-season. Divisions only, \$2.00.

MARIE LEMOINE (Calot $1869 ; 8.5$ ). This is the absolutely indispensable Peony to any collection, large or small. Exquisitely beautiful, massive and solid ivory white, deepening to chamois toward the center and very occassionally tipped with carmine. Invariably a six to seven inch blossom, even under indifferent cultivation. A fragrant free bloomer on extra stiff erect stems and, being a very late white, particularly valuable. Divisions only, $\$ 1.00$.

MARTHA BULLOCH (Brand 1907; 9.1). Late blooming, double, very large, fragrant blossoms of bright rose pink with lighter high lights. Light green, narrow foliage. Tall strong stems. Splendid for exhibition purposes. Divisions only, $\$ 7.50$.

MARY BRAND (Brand 1907; 8.7). This mid-season double Peony is a rich, Indian Lake red showing a few long slender yellow stamens. Twoyear, $\$ 4.50$. One-year, $\$ 3.75$. Divisions, $\$ 3.00$.

MATHILDE DE ROSENECK (Crousse 1883; 7.9). Frankly, this Peony has not sold well. If we changed its name to "Lindy" would we like it better? Of course we would! But why not like it in spite of the name? The soft, rhodonite pink of the outer petals contrasts with the deeper cen. ter and the touch of silver and, here and there a touch of red, on the edges of the petals. Occasional small salmon colored petals scattered throughout blossom. Its fragrance is truly delicious; it opens late, forming a double ball-shaped flower. Two-year, $\$ 1.50$. Divisions, $\$ 1.00$.

MILTON HILL (Richardson; 9.0). Of great size, densely compact and crowded with petals of the finest texture. An exquisite "sea-shell" pink. A late bloomer and undoubtedly one of Richardson's best productions. Divisions only, $\$ 5.00$.

MODELE DE PERFECTION (Crousse 1875; 7.8). This delightfully refreshing flower is a full, compact double form. It is pale rose pink shaded darker in the center and bordered with silvery rose-tipped collar and large guards, forming high globular buds. It is a fragrant, free bloomer. Two-year, $\$ 1.50$. Divisions, $\$ 1.00$.

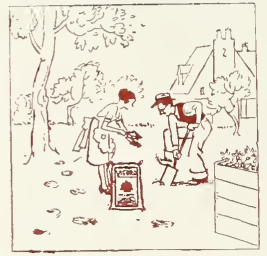

MODESTE GUERIN (Guerin 1845; 7.8). One of the loveliest of them all and of great all-round merit. Very large, full and compact. Superb deep solid tyrian pink. Very free TWEIVE OF ANY ONE VARIETY FOR THE PRICE OF TEN 
flowering and fragrant. Exceptionally rigid stems and very distinctive foliage. Divisions only, $\$ 2.00$.

MONSIEUR BOUCHARLAT AINE (Calot 1868; 7.1). This is one of the beautiful and fragrant mid-season varieties. The full, semi-double flowers are rose-colored with silvery reflections. Two-year, $\$ 1.50$. Divisions, $\$ 1.00$.

MONSIEUR DUPONT (Calot $1872 ; 8.3$ ). Immense, milk-white, beautifully built flower. Central petals prominently splashed with bright acajou red and a few golden stamens discernible at their base. An exceedingly fragrant, free-blooming, late mid-season variety, growing on long stiff upright stems. One of the best whites we have for cutting. A rich and rarely beautiful flower. Divisions only, $\$ 1.00$.

MONSIEUR JULES ELIE (Crousse 1888; 9.2). Immense, globular, very full, double, imbricated bloom on very heavy stems; petalage broad and overlapping, forming one of the most perfect specimens imaginable; color, an ideal, glossy, pale amaranth pink, shading deeper at the base, and the entire blossom covered with a silvery reflex. A vigorous plant, freely blooming, very fragrant. Scarcely rivalled either for cut flowers or for landscape work. Divisions only, $\$ 2.00$.

MONSIEUR MARTIN CAHUZAC (Dessert 1899; 8.8). The darkest red Peony in existence; almost black; a brilliant, dark Bordeaux with metallic reflex. Large flower of fine form with rounded petals; plant erect and ideal habit. An extraordinary flower, raggedly put together and therefore unusually decorative. Two-year, $\$ 6.00$. Divisions, $\$ 4.00$.

OCTAVIE DEMAY (Calot 1867; 8.5). An exquisite, early mid-season flower, large in size and well-formed, with large guard petals. Pale flesh color. with darker shaded center. A fragrant profuse bloomer, on short strong stems. An admirable cut flower. Two-year, \$1.50. Divisions, $\$ 1.00$.

OFFICINALIS RUBRA SUPERBA-An early blooming, non-fragrant red. Divisions only, $\$ 1.00$.

PHILIPPE RIVOIRE (Riviere 1911; 9.2). This very scarce, bright amaranth purple flower, of beautiful form and sturdy growth, is the most fragrant of the red Peonies. Its petals are of unusually glossy texture. No stamens in evidence. Mid-season. Divisions only, $\$ 15.00$.

PIERRE DUCHARTRE (Crousse 1895; 8.2). This is one of the latest bloomers. The flowers are very large and compact with an exquisite fragrance. In color they are a lovely light lilac-pink with a silvery reflex. Divisions only, $\$ 2.00$.

PRESIDENT WILSON (Thurlow 1918; 9.3). Soft amaranth pink, changing to shell-pink as flower opens, guard petals sometimes show crimson markings. Center petals very short, somewhat lacinated, gradually increasing in length outward, producing a cup-like effect. Fragrant. Late. This is generally considered one of the finest varieties for garden or for cut flower. The blooms will last a long time and hold their delicate coloring to the end. Divisions only, $\$ 40.00$.

PRIMEVERE (Lemoine 1907; 8.6). Mid-season, bomb type. Creamy white guards splashed crimson;amber yellow center. Fragrant, fairly compact and flat. Tall and vigorous. The most yellow of all double Peonies. Two-year, \$4.50. One-year, \$3.75. Divisions, \$3.00.

REINE HORTENSE (Calot 1857; 8.7). A delightful pale congo pink with occasional stripings of pomgranite purple. Prominent yellow stamens. The fragrant semi-double rather

TWELTE OF ANY ONE VARIETY FOR THE PRICE OF TEN

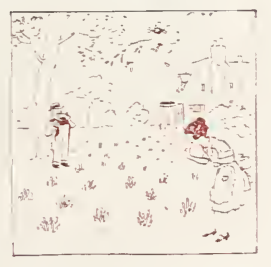



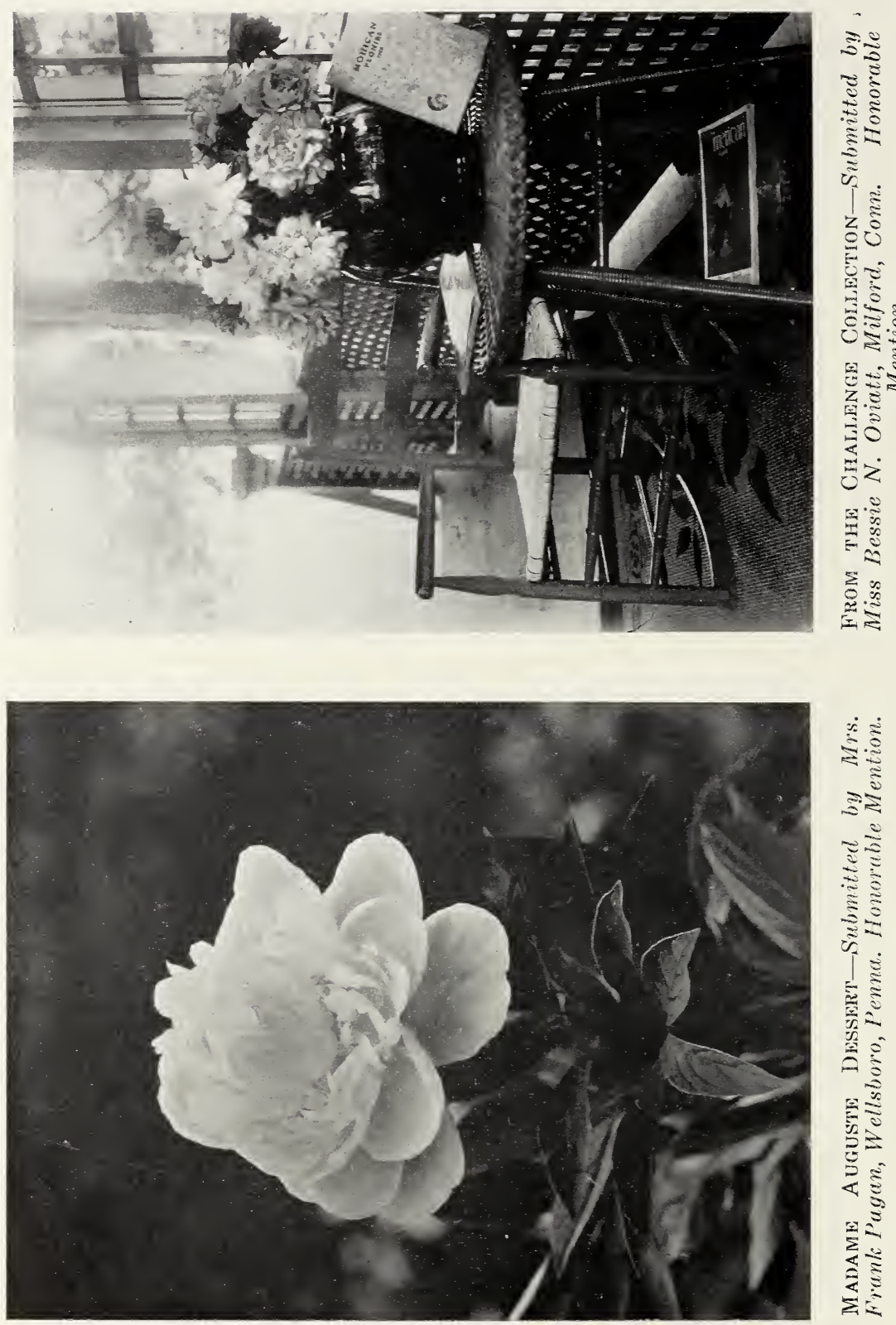

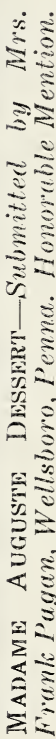




\section{MOHICAN PEONIES}

flat flowers open on tall stems during mid-season. A good cut-flower variety. Two-year, $\$ 2.25$. Divisions, $\$ 1.50$.

RICHARD CARVEL (Brand $1913 ; 8.8$ ). This is one of the few red Peonies that are sweet-scented. Its color is a clear, deep red that does not burn in the sunshine. It is double in form and free blooming. As it blooms early and is an attractive cut-flower variety, it is a desirable addition to any collection. Two-year, $\$ 9.00$. Divisions, $\$ 6.00$.

ROSE SHAYLOR (Shaylor 1920; 9.1). Mid-season, very large double blooms. Pale flesh pink lighter at the edges and shading to creamy yellow at the base of the petals; center petals edged with crimson. Opens broad and flat with hollow center showing richly creamy flesh pink. Slightly fragrant. Tall, strong stems. Divisions only, $\$ 25.00$.

SARAH BERNHARDT (Lemoine 1906; 9.0). So well-known and admired that a description seems unnecessary. As a show flower, only a better Sarah Bernhardt can compete. The large, compact, semi-double flowers of apple-blossom pink, tipped silver, open in late midseason, in clusters carried on strong, vigorous plants. It is gifted with a delicious fragrance and, being a dependable show flower, is naturally a good cut flower. Two-year, $\$ 3.75$ One-year, $\$ 3.10$. Divisions, $\$ 2.50$.

SIMONNE CHEVALIER (Dessert $1902 ; 7.9)$. This large, rose-pink of the crown type, is especially valuable as a free-blooming early cut-flower variety. The stems are long and sturdy, carrying the flowers in clusters, if not disbudded. Two-year, \$2.25. Divisions, $\$ 1.50$.

SOLANGE (Lemoine $1907 ; 9.7)$. This flower is almost pink, almost yellow, but not quite either, deep-

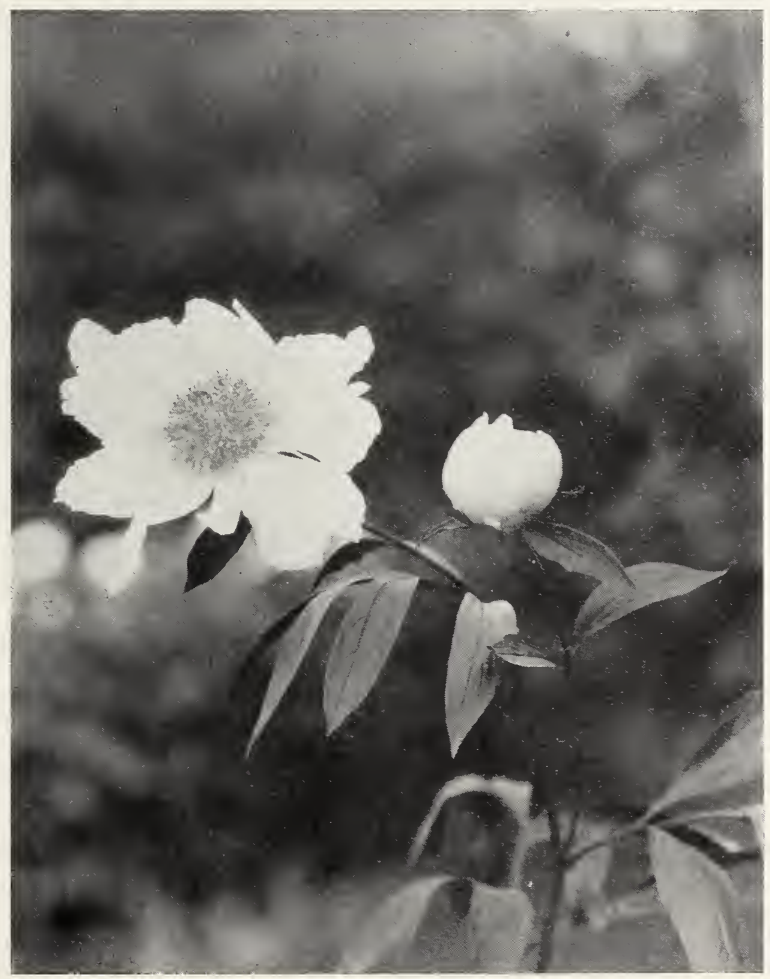

THE BrIDE-Submitted by Mrs. Frank Pagan, Wellsboro, Penna. Honorable Mention.

TWELVE OF ANY ONE VARIETY FOR THE PRICE OF TEN 


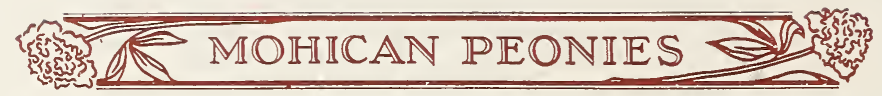

ening to buff at the center. The vigor, size and fragrance of this lovely flower help to give it its place in the trio of the world's best Peonies. Two-year, $\$ 6.00$. Divisions, $\$ 4.00$.

SOLFATARE (Calot 1861; 7.6). One of the few yellow Peonies. The flowers are large, full and fragrant, with white guard petals and center of pale lemon-yellow. A beautiful and distinct variety. Not for sale this year.

SOUVENIR DE L'EXPOSITION DE BORDEAUX (Dessert 1896; 6.4). Medium sized, globular, bomb-shaped; very round and full; color is aster purple which does not fade out under the most glaring sun except at the tips which become tinged with silver. There is nothing else just like it

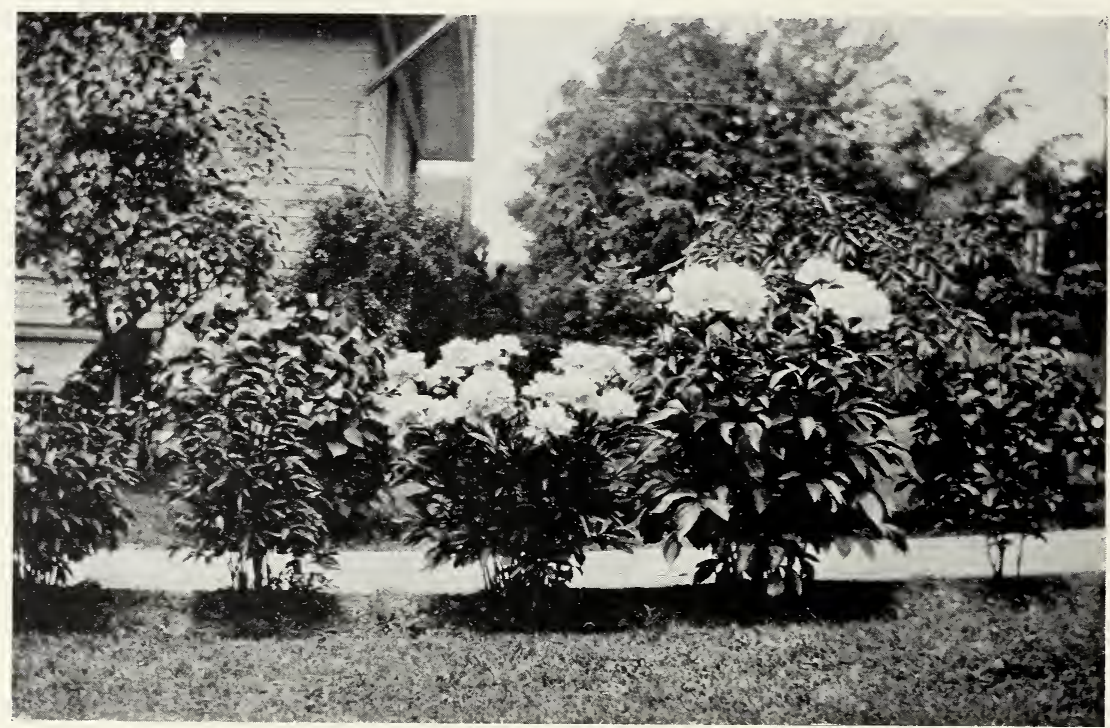

Marie LemoIne-Submitted by Max Engel, Boonton, N. J. Honorable Mention.

in color and it should be included in every collection. A favorite with visitors to our gardens. Medium height, slender-stemmed, profusely blooming. Divisions only, 50c.

SOUVENIR DE LOUIS BIGOT (Dessert 1913; 9.1). Exceptionally large blooms of a very distinctive shade of rich, salmon-pink, with silvery lights, mildly fragrant. This is an extra-fine and scarce mid-season variety. Divisions only, $\$ 7.50$.

THERESE (Dessert $1904 ; 9.8$ ). Many consider this the most beautiful of all Peonies. The flowers are enormous in size, with delicious fragrance, perfect form and fine texture. The plants are vigorous, with large leaves and long stems. The color is an exquisite rose-pink with deep shadings. In spite of its apparent delicacy, it is one of the best varieties for cutting and is a favorite with

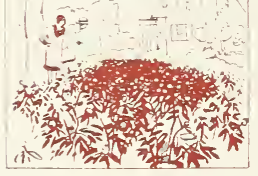
florists. Two-year, $\$ 6.00$. Divisions, $\$ 4.00$.

TWELVE OF ANY ONE VARIETY FOR THE PRICE OF TEN Page Thirty-eight

A Mass of Color 
TOURANGELLE (Dessert 1910; 9.4). Of a delicate opalescent tint that can hardly be described. It is a flower that can be watched lovingly and keenly enjoyed as it unfolds from tight bud to full-blown blossom. Its various depths of delicate pink seem to be caused by the reflection of one petal on another. The transparency of its petals gives it a dreamy loveliness quite unlike any other variety. Divisions only, $\$ 3.50$.

TRIOMPHE DE L'EXPOSITION DE LILLE (Calot 1865; 7.8). The pyramidal shape of this well-constructed Peony distinguishes it from others, except from Modele de Perfection, which it resembles. The compact, double flower is pale rosolane purple with minute touches of a deeper shade. The guard petals are nearly white. It is a fragrant and profuse grower of mid-season and a reliable cut-flower variety. Divisions only, 50c.

UMBELLATA ROSEA (Dessert 1895; 7.4). Considered one of the most valuable of Peonies because it is the earliest to open. The center is flesh color and the guards rose-pink. The double flower is well supported by the strong stems of a vigorous plant; the side buds should be left to bloom for landscape effect. Divisions only, $\$ 1.00$.

VAN DYCK (Crousse 1879; 6.5). Loose, crown type. The cream-white center is flecked with crimson; the guards are pale rose. A fragrant, free bloomer on erect stems. Two-year, \$1.50. Divisions, $\$ 1.00$.

WALTER FAXON (Richardson 1904; 9.3). This is the Peony that people always remember and easily recognize. Its color is a clear, glowing coral pink utterly unlike any other variety. In size, form and vigor this is one of the best. In every way a favorite. Two-year, $\$ 6.00$. Divisions, $\$ 4.00$.

MIXED REDS, PINKS AND WHITES-Peony roots whose tags are lost or whose identity has been lost never find their way back to their companions. We have a limited number of such roots which we offer in 3-5 eye divisions at $50 \mathrm{c}$ each for less than 10 ; at $40 \mathrm{c}$ each for 10 or more; at $35 \mathrm{c}$ each for 25 or more. While they last it is the buyers opportunity.

\section{Single and Japanese Peonies}

\section{ALBIFLORA see "The Bride."}

CLIO (Peterson; 6.5). Large, early, graceful, single, with petals displaying every shade of pink. The strong, well-formed plants with flowers in clusters are very decorative. Two-year, $\$ 3.00$. Divisions, $\$ 2.00$.

KING OF ENGLAND (Kelway $1902 ; 8.6$ ). Japanese, early mid-season. The guards of this very large blossom are dull glossy very dark crimson. The center of incurving buff staminodes is streaked with dark rose pink. Slightly fragrant. Tall slender stems. Divisions only, $\$ 6.00$.

MARIE JACQUIN (Verdier; 8.3). Has a perfection of form that is found in few single Peonies. The pure-white, carefully rounded petals form a cup that closely resembles a waxy white water-lily. Two-year, $\$ 3.00$. Oneyear, $\$ 2.50$. Divisions, $\$ 2.00$.

MIKADO (Barr; 8.6). This is a Japanese Peony whose brilliant rose red petals and yellow center produce a glow of color that resembles in effect a bit of rare old Chinese porcelain. Two-year, \$4.50. One-year, \$3.75. Divisions, $\$ 3.00$.

TWEIVE OF ANY ONE VARIETY FOR THE PRICE OF TEN

Page Thirty-nine

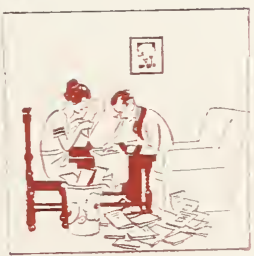

Order Now 
PETITE RENEE (Dessert 1899; 7.6). Approaching Petite Renee one's eye is caught from a distance by its great clusters of loose, large-petalled, rosecolored flowers, with their prominent mustard yellow stamens. Closer examination shows the terminal blossom to be of semi-double form with narrow center petals surrounded by large guards. The lateral flowers are nearly always single and possess a care-free spirit of play in the lightest breeze. This is a charming Peony to plant for landscape effect, since it is not a cut-flower type and should never be disbudded. Divisions only, $\$ 1.50$.

THE BRIDE (Dessert 1902; 8.4). (See illustration on page 37). A large, pure white single Peony with prominent golden stamens. Its fragrant flowers growing in clusters on strong stiff stems and its beautiful foliage make it especially desirable for landscape effect. Two-year, \$3.75. Divisions, $\$ 2.50$.

TOKIO (Japan; 8.9). A splendid landscape variety of good size, on tall strong stems. The broad, well-rounded petals are mallow pink deepening almost to purple on outer edges. Single. Divisions, $\$ 10.00$.

\section{Tree Peonies}

BANSKI-Large semi-double flesh pink flowers. Each, $\$ 4.00$.

TWELVE OF ANY ONE VARIETY FOR THE PRICE OF TEN 



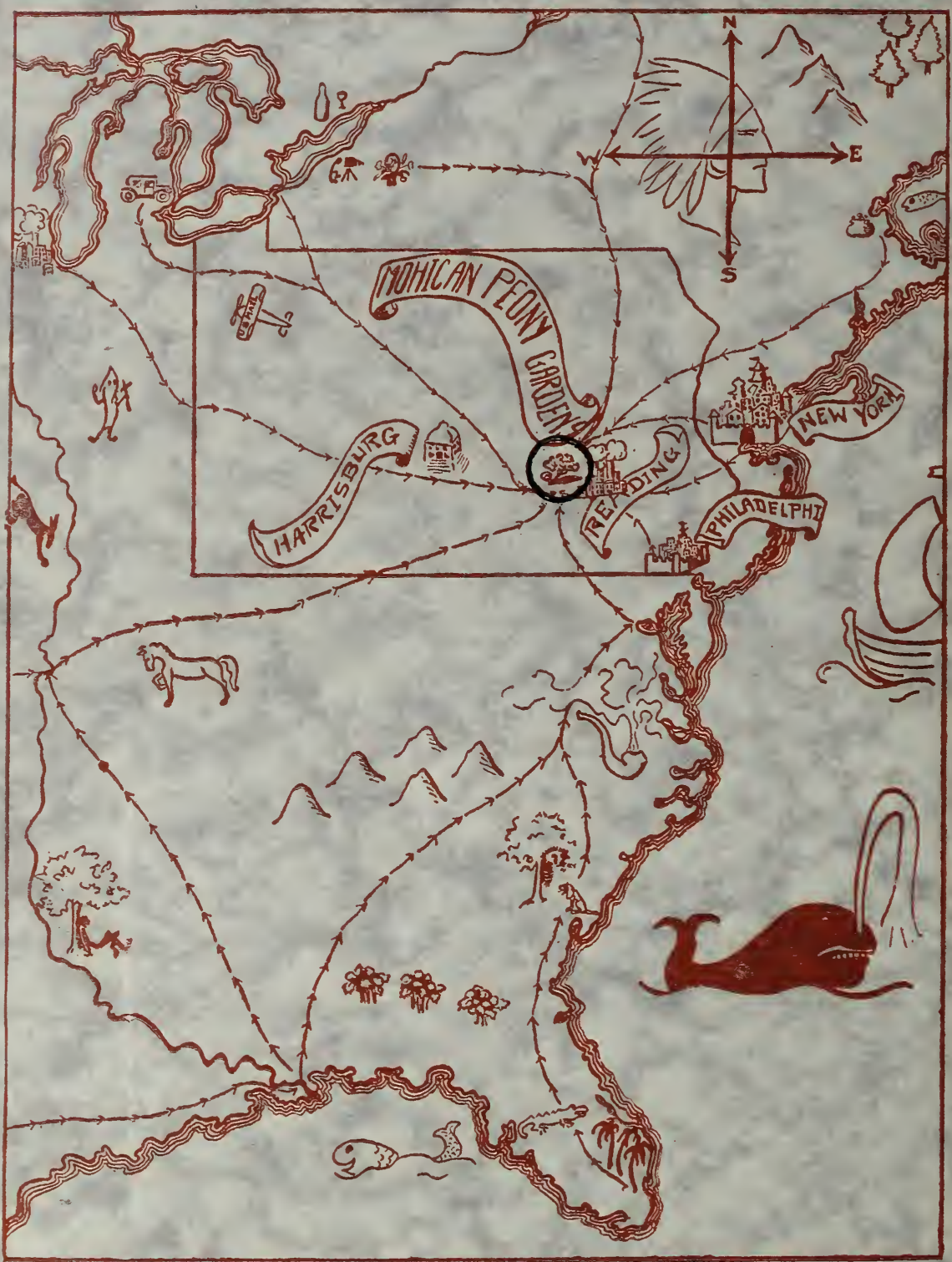

\section{AN INVITATION}

We like to think that our catalog establishes a bond of friendship between us and our customers. This delightful friendship is very real to us and we have tried to make it real to you in this bit of print.

To make the relationship more personal, won't you please find opportunity to visit our gardens this spring when the Peonies are in bloom? Usually during the second and third weeks of June the Gardens are gorgeous. You will be welcome at any time of day or evening-Sundays included.

Printed in U. S. A. 